December 17, 2019

\title{
TOPOLOGY OF SURFACES WITH FINITE WILLMORE ENERGY
}

\author{
JIE ZHOU
}

\begin{abstract}
In this paper, we study the critical case of the Allard regularity theorem. Combining with Reifenberg's topological disk theorem, we get a critical Allard-Reifenberg type regularity theorem. As a main result, we get the topological finiteness for a class of properly immersed surfaces in $\mathbb{R}^{n}$ with finite Willmore energy. Especially, we prove a removability of singularity of multiplicity one surface with finite Willmore energy and a uniqueness theorem of the catenoid under no a priori topological finiteness assumption.
\end{abstract}

\section{Contents}

1. Introduction

2. Lipschitz Approximation

2.1. Preliminaries

2.2. Semi-Reifenberg Condition

2.3. Lipschitz Approximation

3. $C^{\alpha}$-Regularity

4. The Density Identity and Topological Finiteness 23

4.1. The Density Formula 23

4.2. The Density Identity 28

4.3. Topological Finiteness 31

5. Applications 34

5.1. Isolated Singularities 34

5.2. Uniqueness of The Catenoid and Minimal Ends 36

References $\quad 37$

\section{INTRODUCTION}

Assume $\Sigma \subset \mathbb{R}^{n}$ is a properly immersed smooth surface and denote the immersion by $f: \Sigma \rightarrow \mathbb{R}^{n}$. Let $g=f^{*} g_{\mathbb{R}^{n}}$ be the induced metric and $H_{f}=\triangle_{g} f$ be the mean curvature. If $H_{f}=0, f$ is called a minimal immersion and $\Sigma$ is called an immersed minimal surface in $\mathbb{R}^{n}$. One of the most important property for minimal surfaces in $\mathbb{R}^{n}$ is the monotonicity formula, i.e., for $x \in \mathbb{R}^{n}$,

$$
\Theta(x, r)=\frac{\mathcal{H}^{2}\left(B_{r}(x) \cap \Sigma\right)}{\pi r^{2}}
$$

is increasing, where $\mathcal{H}^{2}$ is the two dimensional Hausdorff measure in $\mathbb{R}^{n}$. It implies the density

$$
\Theta(\Sigma, \infty)=\lim _{r \rightarrow+\infty} \Theta(x, r) \in[1, \infty]
$$


of a minimal surface at infinity is well defined. A first important fact about the density of minimal surface is the following corollary of the Allard regularity theorem[1]: if an immersed minimal surface satisfying $\Theta(\Sigma, \infty)<1+\varepsilon$ for $\varepsilon$ sufficient small, then $\Sigma$ is a plane. For $\Theta(\Sigma, \infty)=2$, in the case $n=3$, there are two typical nontrivial examples - the catenoid $\left(x_{1}^{2}+x_{2}^{2}=c h^{2} x_{3}\right)$ and Scherk's singly-periodic surface. They are both embedded minimal surfaces. The catenoid is rotationally

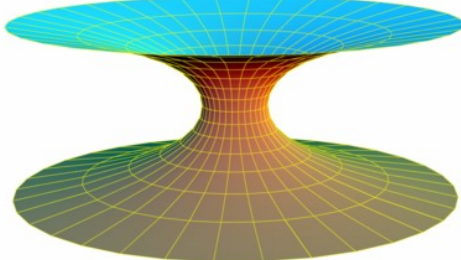

(Catenoid)

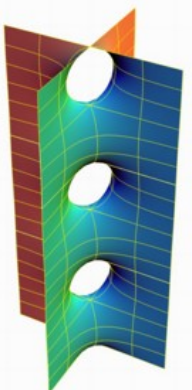

(Scherk's singly periodic surface)

All pictures of minimal surfaces in this paper are taken from wWw. indiana. edu/ minimal.

symmetric, is the simplest minimal surface except for the plane and can be regarded as the fundamental solution of minimal surface equation. The catenoid has finite topology and finite total curvature but Scherk's singly periodic surface has infinite topology and infinite total curvature. And it is found by Karcher[19] that there is a one parameter deformation $\Sigma_{\theta}, \theta \in\left(0, \frac{\pi}{2}\right]$, of Scherk's surface $\Sigma_{\frac{\pi}{2}}$. They are all embedded minimal surfaces with $\Theta\left(\Sigma_{\theta}, \infty\right)=2$ and are also called Scherk's surfaces. Conversely, Meeks and Wolf proved:

Theorem (Meeks-Wolf,[27]). A connected properly immersed minimal surface in $\mathbb{R}^{3}$ with infinite symmetry group and $\Theta(\Sigma, \infty)<3$ is a plane, a catenoid or a Scherk singly-periodic minimal surface $\Sigma_{\theta}, \theta \in\left(0, \frac{\pi}{2}\right]$.

They conjecture the infinite symmetry condition can be removed(see also Conjecture 10 in[25]). For $3 \leq \Theta(\Sigma, \infty)<\infty$, there are not so clear classification, and Meeks and Wolf also conjecture such minimal surfaces admit unique tangent cone at infinity[27, Conjecture 1].

Besides the above uniqueness result of Meeks and Wolf, there are many classical classification theorems for minimal surfaces[29][30][24][23][7][8][9][34][16]. Their common requirement is the minimal surface has finite total curvature, i.e.,

$$
\int_{\Sigma}|A|^{2} d \mathcal{H}^{2}<\infty
$$

where $A$ is the second fundamental form of the surface. Especially, by moving plane method, Schoen[34] proved the only connected complete immersed minimal surface in $\mathbb{R}^{3}$ with finite total curvature and two embedding ends is the catenoid. There is a purely topological description for embedded minimal surface with finite total curvature. A surface is said to have finite topology if it is homeomorphic to a closed surface with finite many points removed. And the number of ends of a properly 
immersed minimal surface is defined by the number of the noncompact connected components of the surface at infinity, i.e.,

$$
e(\Sigma, \infty)=\lim _{r \rightarrow \infty} \tilde{\beta}_{0}\left(\Sigma \cap\left(\mathbb{R}^{n} \backslash B_{r}(0)\right)\right) \in[1, \infty],
$$

where by $\tilde{\beta}_{0}$ we mean the number of noncompact connected components of a topology space. Each such noncompact connected component at infinity is called an end of $\Sigma$. On the one hand, by Huber's result[17], any surface in $\mathbb{R}^{n}$ with finite total curvature must has finite topology. On the other hand, with Meeks and Rosenberg's[26] classification of the complex structure of properly embedded minimal surface with at least two ends, Collin [6] proved a properly embedded minimal surface in $\mathbb{R}^{3}$ with at least two ends has finite total curvature if and only if it has finite topology. In both [26] and [6], the assumption $e(\Sigma, \infty) \geq 2$ is necessary to rule out the helicoid type ends. To distinguish the number of ends is also helpful for understanding Meek's conjecture. The catenoid has two ends. But the "two" tangent planes of Scherk's singly-periodic surface joint together, which forces the surface to possess only one end. So a corollary of Meek's conjecture is that the only connected properly immersed minimal surface in $\mathbb{R}^{3}$ with $\Theta(\Sigma, \infty)<3$ and at least two ends is the catenoid. By the results of Schoen and Collin recalled above, the only gap to the corollary is the topological finiteness of the surface. And the topological finiteness is the main question we care about in this paper:

For a surface immersed in $\mathbb{R}^{n}$ with finite Willmore energy $\int_{\Sigma}|H|^{2} d \mathcal{H}^{2}<\infty$ (or simply, $H=0$ ), when does it have finite topology?

The counterexample of Scherk's singly periodic minimal surface gives some geometric intuition: The number of ends should not be too less with respect to the density. Otherwise, "different" tangent planes at infinity will twist together to shape infinite many genuses. And our answer is:

Theorem 1.1 (Finite Topology). Assume $\Sigma \subset \mathbb{R}^{2+k}$ is a properly immersed open surface with finite Willmore energy, i.e.,

$$
\int_{\Sigma}|H|^{2} d \mathcal{H}^{2}<\infty
$$

If its number of ends is not less than the lower density at infinity, more precisely,

$$
e(\Sigma, \infty)>\Theta_{*}(\Sigma, \infty)-1<+\infty,
$$

then $\Sigma$ has finite topology and finite total curvature and $\Theta(\Sigma, \infty)=e(\Sigma, \infty)$ is an integer number.

By some geometric measure theory argument[21](see Remark 4.8), the assumption $e(\Sigma, \infty)>\Theta_{*}(\Sigma, \infty)-1$ in fact implies $\Sigma$ has exact $e=e(\Sigma, \infty)=\Theta(\Sigma, \infty)$ many ends and each of them has density one at infinity. By the compactness theorem for integral varifolds[1], these ends blow down to planes with multiplicity one. Thus by Leon Simon's theorem on the uniqueness of tangent cone with smooth cross section[41][42, page 269, The paragraph after Theorem 5.7], in the case of

$$
H=0,
$$

each end of $\Sigma$ is a graph over a tangent plane, hence already has finite topology.

$\operatorname{In}[41]$ and [42], by using the variation structure and PDE techniques, especially the monotonicity formula and the 3-circle theorem, Leon Simon established a decay 
estimate around the isolated singularities of solutions for very general variation equations and got the uniqueness of the tangent cone at isolated singular points. Leon Simon also showed[42] the same method works for the tangent cone at infinity. This method is very powerful in analysing the asymptotic behavior of Geometric PDE. For decades, the general method has been applied to many geometric objects including minimal surfaces, harmonic maps, Einstein metrics and corresponding geometric flows. These conclusions imply much more analytic information than the topological finiteness. And we are trying to understand if only caring about the topology, can we get a soft result under looser condition without equation. Theorem 1.1 is the answer. Below we still take the case of $H=0$ to explain our key observation. It will not loss generality.

The idea comes out when we are watching minimal surfaces by the inversion. By combining the monotonicity formulae of a minimal surface $\Sigma$ and its inverted surface $\tilde{\Sigma}$ and a key conformal antisymmetrical invariance we observe(see (37)), we get the following density identity:

$$
\Theta(\tilde{\Sigma}, p)=\frac{1}{16 \pi} \int_{\tilde{\Sigma} \backslash\{p\}}|\tilde{H}|^{2} d \mu_{\tilde{g}}=\Theta(\Sigma, \infty), \quad p \notin \Sigma,
$$

which means the single quantity $\Theta(\Sigma, \infty)$ can control both the Willmore energy $\int_{\tilde{\Sigma} \backslash\{p\}}|\tilde{H}|^{2} d \mu_{\tilde{g}}$ and the local density $\Theta(\tilde{\Sigma}, p)$ of $\tilde{\Sigma}$ at the inverting base point $p$.

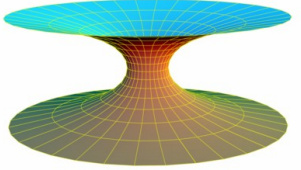

(Catenoid)

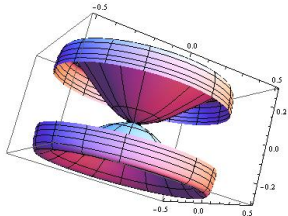

(Part of inverted catenoid)

This implies if we invert only one end with density one, then we will get a varifold with density one at the inverting point and bounded Willmore energy, which is on the border of the classical Allard regularity theorem. Recall the Allard regularity theorem[1] says if an integral $n$-varifold $V=\underline{v}(M, \theta)$ in $B_{r}(0) \subset \mathbb{R}^{n+k}$ satisfies

$$
\Theta^{n}(V, 0)<1+\varepsilon, \quad\left(r^{p-n} \int_{B_{r}(0)}|H|^{p}\right)^{1 / p}<\varepsilon
$$

for some $p>n, \varepsilon$ small and $0 \in \operatorname{spt} V$, then the varifold is a $C^{1, \alpha=1-\frac{n}{p}}$ graph in a small neighborhood of 0 . For a smooth immersion $f: M^{n} \rightarrow \mathbb{R}^{n+k}, H=\triangle_{g} f$. Comparing a varifold to a function, then the generalized mean curvature should be regarded as the weak "Laplacian". In this viewpoint, Allard regularity theorem could be regarded as a geometric nonlinear disturbed version of the $W^{2, p}$ estimates for solutions of linear elliptic equations, combining with the Sobolev embedding theorem $W^{2, p} \hookrightarrow C^{1,1-\frac{n}{p}}$.

\begin{tabular}{|c|c|c|c|c|}
\hline Geometry & smooth manifold & Varifold & weak $H$ & Allard Regularity \\
\hline Analysis & smooth function & Sobolev function & $\triangle_{\text {dist }} f$ & $W^{2, p}$ Esitimate \\
\hline
\end{tabular}

But the mean curvature equation is nonlinear, when getting regularity, one need to do linear approximation first and then use a supercritical index (here $p>n$ ) 
to get an iteration program and then a Campanato type regularity estimate. And now it is in the critical case $p=n=2$. The best expected result is a regularity of type $\left(W^{2,2} \hookrightarrow\right) C^{\alpha}$. By the experience of graphical estimate(See [4, Lemma 2.11] or [5, Lemma 2.4]), the graphical result is always corresponding to a Lipschitz estimate, which seems impossible in our case. So we may not get a $C^{\alpha}$ graph but only get a $C^{\alpha}$ parametrization, which is also enough to show the end is embedded and has finite topology. There is another positive evidence. In [44], Sun and the author proved a properly immersed smooth surface in the unit ball with finite area and small total curvature admits $C^{\alpha}$ parametrization with uniform estimate in some uniform small scale, which can be regarded as a geometric disturbed version of Sobolev's embedding $W^{2,2} \hookrightarrow C^{\alpha}$. This indicates the $C^{\alpha}$ parametrization is hopeful and encourages us to check the original proof of Allard regularity theorem in the critical case. It turns out there is no difficulty in getting the Lipschitz approximation[40, Section 20] from Leon Simon's monotonicity formula[38], but it is impossible to run the iteration program to get a decay of the tilt-excess[40, Section 22].

Fortunately, there is a well developed criteria for the $C^{\alpha}$ regularity of a closed set in $\mathbb{R}^{n}$. That is Reifenberg's topological disk theorem[31][28][43], whose proof contains a geometric iteration program. Reifenberg's theorem has been established in 1960. Recent twenty years, many mathematicians used the method to research the regularity of both Ricci limit spaces and Radon measures. Let us refer [2, Appendix] [11][45][12, section 7][13] for readers who are interested in related topics. Especially, Paolini proved[45] the $C^{\alpha}$ regularity for minimal boundaries in $\mathbb{R}^{n}$ with mean curvature in $L^{n}$. Similarly, in our critical case, when combining with the Lipschitz approximation, we can check Reifenberg's condition. As a result, we get the $C^{\alpha}$-regularity for rectifiable 2 -varifold with square integrable generalized mean curvature at those points with density close to one. See Theorem 3.1 for precise statement.

As an application of Theorem 1.1, we studied isolated singularities for properly immersed surfaces with finite Willmore energy. We get the removability of such singularities under the assumption of density less than two. We do not assume the surface have finite topology or finite total curvature a priori. See Corollary 5.2 for details. As corollaries of Theorem 1.1, we also give a simple proof of the uniqueness of the catenoid(see Corollary 5.4) and analysis the structure of minimal ends in $\mathbb{R}^{2+k}$ with multiplicity less than two.

This paper is organized as following. In section 2, we prove the Lipschitz approximation theorem. In section 3 , we check the Reifenberg condition and complete the proof of the $C^{\alpha}$ regularity. In section 4 , we deduce the density identity of inverting minimal surface and apply the $C^{\alpha}$ regularity theorem to ends with density less than two to get the main theorem of this paper. In section 5 , we give the two applications.

\section{LipsChitz Approximation}

In this section, we check out the Lipschitz approximation theorem in the critical case. It is the first step of proving the Allard regularity theorem and many of the ideas are similar to those of [40](see also [39, section 5.2, 5.4, 5.5, 5.6]), except for a careful analysis involving the remainder term of Leon Simon's monotonicity identity (1) in the proof Lemma 2.10 and some other details. We also focus on the 
semi-Reifenberg condition (7), which is essential for the proof of the $C^{\alpha}$ regularity theorem in section 3 .

For a rectifiable 2 -varifold $V=\underline{v}(\Sigma, \theta)$ in an open set $U \subset \mathbb{R}^{n}$, we always denote the corresponding Radon measure by $\mu=\mu_{V}=\mathcal{H}^{2}\left\llcorner\theta\right.$, i.e, for any Borel set $A \subset \mathbb{R}^{n}$,

$$
\mu(A)=\mu_{V}(A)=\int_{A \cap \Sigma} \theta d \mathcal{H}^{2} .
$$

The following is the main result of this section-the Lipschitz approximation theorem.

Theorem 2.1 (Lipschitz Approximation for 2-varifold). Assume $V=\underline{v}(\Sigma, \theta)$ is a rectifiable 2-varifold in $U \supset B_{\rho}(0) \subset \mathbb{R}^{2+k}$ with generalized mean curvature $H \in L^{2}\left(d \mu_{V}\right), 0 \in \operatorname{spt} V$ and $\theta \geq 1$ for $\mu_{V}-$ a.e. $x \in U$. Then there exists small $\delta_{6}\left(=\frac{1}{2^{1688} k^{40}}\right)$ such that for any $\bar{\delta} \leq \delta_{6}$ if

$$
\frac{\mu_{V}\left(B_{\rho}(0)\right)}{\pi \rho^{2}} \leq 1+\delta \text { and } \int_{B_{\rho}(0)}|H|^{2} \leq \delta
$$

then for any $\xi \in B_{\frac{1}{2} \delta^{\frac{1}{2}} \rho}(0)$ and $\sigma \in\left(0, \frac{1}{2^{16}} \delta^{\frac{1}{2}} \rho\right)$, there exist $T=T(\xi, \sigma) \in G_{2+k, 2}(\mathbb{R})$ passing through $\xi$ and a Lipschitz function

$$
f=\left(f^{1}, f^{2}, \ldots f^{k}\right): B_{\sigma}(\xi) \cap T \rightarrow \mathbb{R}^{k}:=T^{\perp}
$$

with

i) $\operatorname{Lipf} \leq \delta^{\frac{1}{40}}$

ii) $\sup _{x \in B_{\sigma}(\xi)}|f(x)| \leq \delta^{\frac{1}{40}} \sigma$,

$\sup _{x \in B_{\sigma}(\xi) \cap s p t \mu_{V}}|q(x)| \leq \delta^{\frac{1}{40}} \sigma$,

iv) $\mathcal{H}^{2}\left((\operatorname{graph} f \backslash \operatorname{spt} V) \cap B_{\sigma}(\xi)\right)+\mu_{V}\left(B_{\sigma}(\xi) \backslash\right.$ graphf $) \leq 2^{83} \delta^{\frac{1}{16}} \pi \sigma^{2}$,

where $q: \mathbb{R}^{2+k} \rightarrow T^{\perp}$ is the orthogonal projection.

\subsection{Preliminaries.}

We begin with some preliminaries: the monotonicity formula and its corollaries.

Lemma 2.2. [38][22] Assume $V=\underline{v}(\Sigma, \theta)$ is a rectifiable 2-varifold in an open set $U \subset \mathbb{R}^{2+k}$ with generalized mean curvature $H \in L^{2}(d \mu)$. Then, for any $x \in \mathbb{R}^{2+k}$, and $0<\sigma<\rho<\infty$ with $B_{\rho}(x) \subset U$,

$$
\begin{aligned}
\frac{\mu\left(B_{\sigma}(x)\right)}{\sigma^{2}}=\frac{\mu\left(B_{\rho}(x)\right)}{\rho^{2}} & +\frac{1}{16} \int_{B_{\rho}(x) \backslash B_{\sigma}(x)}|H|^{2} d \mu-\int_{B_{\rho} \backslash B_{\sigma}}\left|\frac{\nabla^{\perp} r}{r}+\frac{H}{4}\right|^{2} d \mu \\
& +\frac{1}{2 \rho^{2}} \int_{B_{\rho}(x)} r\left\langle\nabla^{\perp} r, H\right\rangle d \mu-\frac{1}{2 \sigma^{2}} \int_{B_{\sigma}(x)} r\left\langle\nabla^{\perp} r, H\right\rangle d \mu,
\end{aligned}
$$

Where $r=r_{x}=|\cdot-x|$. Moreover, for any $\delta \leq 1$, we have

$$
\frac{\mu\left(B_{\sigma}(x)\right)}{\sigma^{2}} \leq(1+\delta) \frac{\mu\left(B_{\rho}(x)\right)}{\rho^{2}}+\frac{1}{2 \delta} \int_{B_{\rho}(x)}|H|^{2} d \mu .
$$


Corollary 2.3. Assume $V=\underline{v}(\Sigma, \theta)$ is a rectifiable 2-varifold in an open set $U \subset \mathbb{R}^{2+k}$ with generalized mean curvature $H \in L^{2}(d \mu)$ and $B_{\rho}(0) \subset U$. If $\int_{B_{\rho}(0)}|H|^{2} d \mu+\mu\left(B_{\rho}(0)\right)<\infty$ and $\theta(x) \geq 1$, for $\mu-$ a.e. $x \in$ spt $\mu$. Then

$$
\Theta(x)=\lim _{\tau \rightarrow 0} \frac{\mu\left(B_{\tau}(x)\right)}{\pi \tau^{2}}
$$

is well-defined in $\breve{B}_{\rho}(0)$ and is upper semi-continuous. Moreover, for any $x \in \breve{B}_{\rho}(0)$,

$$
\Theta(x) \geq 1 \text {. }
$$

Proof. See [22, Appendix]

The following corollary is prepared for section 4. For simplicity, we will omit the measure notation $d \mu$ under the integral from now on.

Corollary 2.4. Assume $V=\underline{v}(\Sigma, \theta)$ is a rectifiable 2-varifold in $\mathbb{R}^{n}$ with generalized mean curvature $H \in L^{2}\left(\mathbb{R}^{n}, d \mu_{V}\right)$. Then, for any $x \in \mathbb{R}^{n}$,

$$
\Theta^{*}(V, \infty):=\limsup _{r \rightarrow \infty} \frac{\mu_{V}\left(B_{r}(x)\right)}{\pi r^{2}}<+\infty
$$

if and only if

$$
\Theta_{*}(V, \infty):=\liminf _{r \rightarrow \infty} \frac{\mu_{V}\left(B_{r}(x)\right)}{\pi r^{2}}<+\infty
$$

if and only if

$$
\int_{\mathbb{R}^{n}}\left|\frac{\nabla^{\perp} r_{x}}{r_{x}}\right|^{2}<\infty
$$

Moreover, if one of the above condition holds, then for any $\rho \in(0, \infty)$,

$$
\frac{\mu_{V}\left(B_{\rho}(x)\right)}{\pi \rho^{2}} \leq 9 \Theta_{*}(V, \infty)+\frac{59}{16 \pi} \int_{\mathbb{R}^{n}}|H|^{2} .
$$

Proof. For simplicity, we denote $r_{x}=|\cdot-x|$ by $r$. Since

$$
\left|\frac{1}{2 \sigma^{2}} \int_{B_{\sigma}(x)} r\left\langle\nabla^{\perp} r, H\right\rangle\right| \leq \frac{\left(\mu\left(B_{\sigma}(x)\right)\right)^{\frac{1}{2}}}{2 \sigma}\|H\|_{L^{2}\left(B_{\sigma}(x)\right)} \rightarrow 0,
$$

letting $\sigma \rightarrow 0$ in the monotonicity formula (1), we get

$$
\int_{B_{\rho}(x)}\left|\frac{\nabla^{\perp} r}{r}+\frac{H}{4}\right|^{2}-\frac{1}{2 \rho^{2}} \int_{B_{\rho}(x)} r\left\langle\nabla^{\perp} r, H\right\rangle=\frac{\mu_{V}\left(B_{\rho}(x)\right)}{\rho^{2}}-\pi \Theta(x)+\frac{1}{16} \int_{B_{\rho}(x)}|H|^{2},
$$

which implies $\int_{B_{\rho}(x)}\left|\frac{\nabla^{\perp} r}{r}\right|^{2}<+\infty$. Note

$$
\frac{1}{2} \int_{B_{\rho}(x)}\left|\frac{\nabla^{\perp} r}{r}\right|^{2}-\int_{B_{\rho}(x)}\left|\frac{H}{4}\right|^{2} \leq \int_{B_{\rho}(x)}\left|\frac{\nabla^{\perp} r}{r}+\frac{H}{4}\right|^{2} \leq 2 \int_{B_{\rho}(x)}\left(\left|\frac{\nabla^{\perp} r}{r}\right|^{2}+\left|\frac{H}{4}\right|^{2}\right)
$$

and

We know

$$
\left|\frac{1}{2 \rho^{2}} \int_{B_{\rho}(x)} r\left\langle\nabla^{\perp} r, H\right\rangle\right| \leq \frac{1}{4} \int_{B_{\rho}(x)}\left|\frac{\nabla^{\perp} r}{r}\right|^{2}+\frac{1}{4} \int_{B_{\rho}(x)}|H|^{2} .
$$

$$
\frac{1}{4} \int_{B_{\rho}(x)}\left|\frac{\nabla^{\perp} r}{r}\right|^{2}-\frac{3}{8} \int_{B_{\rho}(x)}|H|^{2} \leq \frac{\mu_{V}\left(B_{\rho}(x)\right)}{\rho^{2}}-\pi \Theta(x)
$$




$$
\leq \frac{9}{4} \int_{B_{\rho}(x)}\left|\frac{\nabla^{\perp} r}{r}\right|^{2}+\frac{5}{16} \int_{B_{\rho}(x)}|H|^{2} .
$$

Letting $\rho \rightarrow \infty$, we get

$$
\begin{aligned}
\frac{1}{4} \int_{\mathbb{R}^{n}}\left|\frac{\nabla^{\perp} r}{r}\right|^{2}-\frac{3}{8} \int_{\mathbb{R}^{n}}|H|^{2} & \leq \pi\left(\Theta_{*}(V, \infty)-\Theta(x)\right) \\
& \leq \pi\left(\Theta^{*}(V, \infty)-\Theta(x)\right) \\
& \leq \frac{9}{4} \int_{\mathbb{R}^{n}}\left|\frac{\nabla^{\perp} r}{r}\right|^{2}+\frac{5}{16} \int_{\mathbb{R}^{n}}|H|^{2} .
\end{aligned}
$$

Finally, combining the last two lines we get

$$
\frac{\mu\left(B_{\rho}(x)\right.}{\pi \rho^{2}} \leq 9 \Theta_{*}(V, \infty)+\frac{59}{16 \pi} \int_{\mathbb{R}^{n}}|H|^{2} .
$$

\subsection{Semi-Reifenberg Condition.}

In the proof of the Allard regularity theorem, the following non-dimensional(scaling invariant) quantity $E(\xi, \rho, T)$ plays an important role.

Definition 2.5. Assume $V=\underline{v}(\Sigma, \theta)$ is a rectifiable 2 -varifold in $\mathbb{R}^{2+k}$ and $B_{\rho}(\xi) \subset$ $U$. Denote $\mu=\mu_{V}$. For any 2-plane $T$ in $\mathbb{R}^{2+k}$, the tilt-Excess $E(\xi, \rho, T)$ is defined by

$$
E(\xi, \rho, T):=\rho^{-2} \int_{B_{\rho}(\xi)}\left|p_{T_{x} \Sigma-p_{T}}\right|^{2} d \mu,
$$

where $T_{x} \Sigma$ is the approximate tangent plane of the varifold $V$ at $x \in \operatorname{spt} \mu$ and $p_{T}$ and $p_{T_{x} \Sigma}$ are orthogonal projection to $T$ and $T_{x} \Sigma$ respectively.

The tilt-excess measures the mean oscillation of the approximate tangent space( Gaussian map) of the varifold in the ball $B_{\rho}(\xi)$. The oscillation behavior of the tangent spaces are always relating to the regularity of the geometric objects at different levels. For example, the $C^{1, \alpha}$ regularity occurred in the Allard regularity theorem owes to the decay of tilt-excess. Stephen Semmes proved [35][36][37] the Lipschitz regulairty for hypersurfaces in $\mathbb{R}^{n+1}$ with Gaussian maps small BMO. And Reifenberg's topological disk theorem, the key to the $C^{\alpha}$ regularity, is also established on some oscillation condition-the Reifenberg condition (5).

Theorem 2.6 (Reifenberg). [31][28][43] For integers $m, k>0$ and $\alpha>0$, there exists $\varepsilon=\varepsilon(m, k, \alpha)>0$ such that for any closed set $S \subset \mathbb{R}^{m+k}$ with $0 \in S$, if for any $y \in S \cap B_{1}(0)$ and $\rho \in(0,1]$, there exists an $m$-dimensional plane $L_{y, \rho} \subset \mathbb{R}^{m+k}$ passing through $y$ such that

$$
d_{\mathcal{H}}\left(S \cap B_{\rho}(y), L_{y, \rho} \cap B_{\rho}(y)\right) \leq \varepsilon \rho,
$$

then $S \cap B_{1}$ is homeomorphic to the unit ball $B_{1}^{m}(0) \subset \mathbb{R}^{m}$. More precisely, there exist closed set $M \subset \mathbb{R}^{m+k}$ and $m$-dimensional subspace $T_{0} \subset \mathbb{R}^{m+k}$ and a homeomorphism $\tau: T_{0} \rightarrow M$ such that $M \cap B_{1}=S \cap B_{1}$, both $\tau, \tau^{-1} \in C^{\alpha}$ and

$$
|\tau(x)-x| \leq C(m, k) \varepsilon, \forall x \in T_{0} \quad \text { and } \quad \tau(x)=x, \forall x \in T_{0} \backslash B_{2} .
$$

The condition (5) is called the Reifenberg condition. In this subsection, we establish the tilt-excess estimate. By the way, we note the process in fact implies half of the Reifenberg condition, we call it semi-Reifenberg condition (7). 
By noting the integrand in the tilt-excess is just the gradient of the position function, the tilt-excess estimate can be reduced to some " $L^{2}$-estimate" by integral gradient estimate of the generalized mean curvature equation.

Lemma 2.7. Assume $V=\underline{v}(\Sigma, \theta)$ is a rectifiable 2-varifold in an open set $U \subset$ $\mathbb{R}^{2+k}$ with generalized mean curvature $H \in L^{2}(d \mu)$ for $\mu=\mu_{V}$ and $B_{\rho}(\xi) \subset U$. Then, for any 2-plane $T$ in $\mathbb{R}^{2+k}$,

$$
E\left(\xi, \frac{\rho}{2}, T\right) \leq 4 \int_{B_{\rho}(\xi)}|H|^{2}+592 \rho^{-2} \int_{B_{\rho}(\xi)}\left(\frac{d(x, T)}{\rho}\right)^{2} d \mu .
$$

Proof. Take coordinates of $\mathbb{R}^{2+k}$ such that $T=\operatorname{span}\left\{\left(x^{1}, x^{2}, 0, \ldots, 0\right)\right\}$ and $T^{\perp}=$ $\operatorname{span}\left\{X^{\prime}=\left(0,0, x^{3}, x^{4}, \ldots x^{2+k}\right)\right\}$. Then, an observation is

$$
\frac{1}{2}\left|p_{T_{x} \Sigma-p_{T}}\right|^{2}=\Sigma_{j=1}^{k}\left|\nabla^{\Sigma} x^{2+j}\right|^{2}=\operatorname{div}^{\Sigma} X^{\prime}
$$

So, to estimate $E(\xi, \rho, T)=2 \rho^{-2} \int_{B_{\rho}(\xi)} \Sigma_{j=1}^{k}\left|\nabla^{\Sigma} x^{2+j}\right|^{2} d \mu$ is equal to give an integral gradient estimate of the generalized mean curvature equation

$$
\int \operatorname{div}^{\Sigma} X=-\int X \cdot \vec{H}
$$

For details, see [40, Lemma 22.2]

The above lemma reduces the tilt-excess estimate to the " $L^{2}$ - estimate" of the form $\rho^{-2} \int_{B_{\rho}(\xi)}\left(\frac{d(x, T)}{\rho}\right)^{2} d \mu$, whose estimate can be seen as an integral version of half of the Reifenberg condition. The following lemma gives a point-wise semiReifenberg condition, which implies the tilt-excess estimate.

Lemma 2.8 (Semi - Reifenberg Condition). Assume $V=\underline{v}(\Sigma, \theta)$ is a rectifiable 2-varifold in $U \supset B_{\rho}(0) \subset \mathbb{R}^{2+k}$ with generalized mean curvature $H \in L^{2}(d \mu)$, $0 \in \operatorname{spt} V$ and $\theta \geq 1$ for $\mu_{V}-$ a.e. $x \in U$. If for some $\delta \leq 2^{-4}$,

$$
\frac{\mu_{V} B_{\rho}(0)}{\pi \rho^{2}} \leq 1+\delta \text { and } \int_{B_{\rho}(0)}|H|^{2} \leq \delta
$$

then for $\forall \xi \in \operatorname{spt}_{V} \cap B_{\frac{1}{2} \delta^{\frac{1}{2}} \rho}(0)$ and $\forall \sigma \leq \frac{1}{2} \delta^{\frac{1}{2}} \rho$, there exists a $T=T(\xi, \sigma)$ passing through $\xi$, such that

$$
\sigma^{-1} \sup _{x \in \operatorname{spt} \mu_{V} \cap B_{\sigma}(\xi)} d(x, T) \leq 2^{13} \delta^{1 / 16}
$$

Proof. Step 3.1 Volume ratio estimate. For $\forall \xi \in B_{\delta^{\frac{1}{2}} \rho}(0)$ and $\forall \sigma \in\left(0,\left(1-\delta^{\frac{1}{2}}\right) \rho\right)$, we have

$$
\frac{\mu\left(B_{\sigma}(\xi)\right)}{\pi \sigma^{2}} \leq 1+36 \delta^{\frac{1}{2}}
$$

Moreover, if $\xi \in \operatorname{spt} \mu_{V}$, then

$$
\frac{\mu\left(B_{\sigma}(\xi)\right)}{\pi \sigma^{2}} \geq 1-2 \delta^{\frac{1}{2}} .
$$

In fact, take $\beta=\delta^{\frac{1}{2}} \leq \frac{1}{2}$ and $\delta_{0}=\delta^{\frac{1}{2}}$. Then by the monotonicity formula (2), we know

$$
\frac{\mu\left(B_{\sigma}(\xi)\right)}{\pi \sigma^{2}} \leq\left(1+\delta_{0}\right) \frac{\mu\left(B_{\rho-\beta \rho}\right)(\xi)}{\pi(\rho-\beta \rho)^{2}}+\frac{1}{2 \pi \delta_{0}} \int_{B_{\rho-\beta \rho}(\xi)}|H|^{2} \leq 1+36 \delta^{\frac{1}{2}}
$$


On the other hand, for $\xi \in \operatorname{spt} \mu_{V},(2)$ and Corollary 2.3 imply

$$
1 \leq\left(1+\delta_{0}\right) \frac{\mu\left(B_{\sigma}(\xi)\right)}{\pi \sigma^{2}}+\frac{1}{2 \pi \delta_{0}} \int_{B_{\sigma}(\xi)}|H|^{2} \leq\left(1+\delta_{0}\right) \frac{\mu\left(B_{\sigma}(\xi)\right)}{\pi \sigma^{2}}+\frac{\delta}{2 \delta_{0}} .
$$

Thus

$$
\frac{\mu\left(B_{\sigma}(\xi)\right)}{\pi \sigma^{2}} \geq \frac{1-\frac{\delta^{\frac{1}{2}}}{2}}{1+\delta^{\frac{1}{2}}} \geq 1-2 \delta^{\frac{1}{2}} .
$$

Step 3.2 For $\forall \xi \in \operatorname{spt}_{V} \cap B_{\beta \rho}(0)$ and small $\sigma$, the goal is to find $T=T(\xi, \sigma)$ such that

$$
\sigma^{-1} \sup \left\{d(x, T): d \in \operatorname{spt} \mu_{V} \cap B_{\sigma}(\xi)\right\} \text { small . }
$$

It is not easy to get the point estimate directly. So, in the spirit of Chebyshev inequality, we estimate the mean integral value in a small neighborhood of $\xi$. More precisely, for small $\alpha$ (to be determined) and $y \in \operatorname{spt} \mu_{V} \cap B_{\alpha \sigma}(\xi)$, denote $T_{y}$ to be the translation of the approximate tangent space of $\Sigma$ at $y$ (which exists for $\mu$-almost $y$ since $V$ is rectifiable) such that $T_{y} \ni y$. Then $d\left(x, T_{y}\right)=\left|p_{T_{y}}^{\perp}(x-y)\right|$ measures how close $x \in \operatorname{spt} \mu_{V} \cap B_{\sigma}(\xi)$ is to $T_{y}$. Consider the mean integral

$$
\begin{aligned}
\bar{J} & =\frac{1}{(\alpha \sigma)^{2}} \int_{B_{\alpha \sigma}(\xi)} \frac{1}{\sigma^{2}} \int_{B_{\sigma}(\xi)} \frac{d^{2}\left(x, T_{y}\right)}{\sigma^{2}} d \mu(x) d \mu(y) \\
& \leq \frac{1}{\sigma^{2}} \int_{B_{\sigma}(\xi)} \frac{1}{(\alpha \sigma)^{2}} \int_{B_{(\alpha+1) \sigma}(x)} \frac{d^{2}\left(x, T_{y}\right)}{\sigma^{2}} d \mu(y) d \mu(x) .
\end{aligned}
$$

For fixed $x$ and $r_{x}(y)=|y-x|$, note

$$
\left|\nabla^{\perp} r_{x}(y)\right|=r_{x}^{-1}(y)\left|p_{T_{y}}^{\perp}(y-x)\right|=r_{x}^{-1}(y) d\left(x, T_{y}\right) .
$$

So

$$
\begin{aligned}
& \bar{J} \leq \frac{1}{\sigma^{2}} \int_{B_{\sigma}(\xi)} \frac{1}{(\alpha \sigma)^{2}} \int_{B_{(\alpha+1) \sigma}(x)} \frac{r_{x}^{4}}{\sigma^{2}} \frac{\left|\nabla^{\perp} r_{x}\right|^{2}}{r_{x}^{2}} d \mu(y) d \mu(x) \\
& =\frac{(1+\alpha)^{4}}{\alpha^{2} \sigma^{2}} \int_{B_{\sigma}(\xi)} \int_{B_{(\alpha+1) \sigma}(x)} \frac{\left|\nabla^{\perp} r_{x}\right|^{2}}{r_{x}^{2}} d \mu(y) \quad d \mu(x)
\end{aligned}
$$

Note $\Theta(x) \geq 1$ for $x \in \operatorname{spt} \mu_{V}$ and

$$
\lim _{\sigma_{1} \rightarrow 0}\left|\frac{1}{\sigma_{1}^{2}} \int_{B_{\sigma_{1}}(x)} r_{x}\left\langle\nabla^{\perp} r_{x}, H\right\rangle\right| \leq \lim _{\sigma_{1} \rightarrow 0}\left(\int_{B_{\sigma_{1}}(x)}|H|^{2}\right)^{\frac{1}{2}}\left(\frac{\mu\left(B_{\sigma_{1}}(x)\right)}{\sigma_{1}^{2}}\right)^{\frac{1}{2}}=0 .
$$

Taking $\rho_{1}=(1+\alpha) \sigma$, using the monotonicity formula (1) for $0<\sigma_{1}<\rho_{1}$ and then letting $\sigma_{1} \rightarrow 0$, we get

$$
\int_{B_{\rho_{1}}(x)}\left|\frac{\nabla^{\perp} r}{r}+\frac{H}{4}\right|^{2} \leq\left(\frac{\mu\left(B_{\rho_{1}}(x)\right)}{\rho_{1}^{2}}-\pi\right)+\frac{1}{16} \int_{B_{\rho_{1}}(x)}|H|^{2}+\frac{1}{2 \rho_{1}} \int_{B_{\rho_{1}}(x)}|H| .
$$

Taking $\xi \in \operatorname{spt}_{V} \cap B_{\frac{1}{2} \delta^{\frac{1}{2}} \rho}(0), \alpha \leq 1$ and $\sigma \leq \frac{1}{2} \delta^{\frac{1}{2}} \rho$, then $x \in B_{\sigma}(\xi) \in B_{\delta^{\frac{1}{2}} \rho}(0)$ and $(1+\alpha) \sigma<\left(1-\delta^{\frac{1}{2}}\right) \rho$. So, by (8), we get

$$
K(x) \leq \int_{B_{\rho_{1}}(x)} \frac{\left|\nabla^{\perp} r\right|^{2}}{r^{2}} d \mu(y) \leq 2 \int_{B_{\rho_{1}}(x)}\left|\frac{\nabla^{\perp} r}{r}+\frac{H}{4}\right|^{2}+2 \int_{B_{\rho_{1}}(x)}\left|\frac{H}{4}\right|^{2}
$$




$$
\begin{aligned}
& \leq 2\left[\left(\frac{\mu\left(B_{\rho_{1}}(x)\right)}{\rho_{1}^{2}}-\pi\right)+\frac{2}{16} \int_{B_{\rho_{1}}(x)}|H|^{2}+\frac{1}{2}\left(\int_{B_{\rho_{1}}(x)}|H|^{2}\right)\left(\frac{\mu\left(B_{\rho_{1}}(x)\right)}{\rho_{1}}\right)^{\frac{1}{2}}\right] \\
& \leq 80 \pi \delta^{\frac{1}{2}}
\end{aligned}
$$

and

$$
\bar{J} \leq \frac{2^{4} \cdot 80 \pi \delta^{\frac{1}{2}} \mu\left(B_{\sigma}(\xi)\right)}{\alpha^{2} \sigma^{2}} \leq \frac{2^{12} \pi \delta^{\frac{1}{2}}}{\alpha^{2}}=2^{12} \pi \delta^{\frac{1}{4}}\left(\text { take } \alpha=\delta^{\frac{1}{8}}\right) .
$$

Thus by Chebyshev's inequality, there exists $y \in B_{\alpha \sigma}(\xi)$, such that

$$
\frac{1}{\sigma^{2}} \int_{B_{\sigma}(\xi)} \frac{d^{2}\left(x, T_{y}\right)}{\sigma^{2}} d \mu(x) \leq 2^{12} \delta^{\frac{1}{4}} /\left(1-2 \delta^{\frac{1}{2}}\right) \leq 2^{13} \delta^{\frac{1}{4}}\left(\text { take } \delta \leq 2^{-4}\right) .
$$

So if we denote $T=T_{y}+\xi-y$, then $d^{2}(x, T) \leq 2 d^{2}\left(x, T_{y}\right)+2 \alpha^{2} \sigma^{2}$. Thus

$$
\frac{1}{\sigma^{2}} \int_{B_{\sigma}(\xi)} \frac{d^{2}(x, T)}{\sigma^{2}} d \mu(x) \leq 2^{14} \delta^{\frac{1}{4}}+2 \delta^{\frac{1}{4}}\left(\pi+32 \pi \delta^{\frac{1}{2}}\right) \leq 2^{15} \delta^{\frac{1}{4}} .
$$

And by Lemma 2.7, we know that

$$
E\left(\xi, \frac{\sigma}{2}, T(\xi, \sigma)\right) \leq 4 \delta+592 \cdot 2^{15} \delta^{\frac{1}{4}} \leq 2^{25} \delta^{\frac{1}{4}} .
$$

Up to now, we have established the tilt-excess estimate:

Corollary 2.9. under the condition of Lemma 2.8, for $\delta \leq 2^{-4}, \xi \in B_{\frac{1}{2} \delta^{\frac{1}{2}} \rho}(0)$ and $\sigma \leq \frac{1}{2} \delta^{\frac{1}{2}} \rho$, there exists $T=T(\xi, \sigma)$ such that

$$
E(\xi, \sigma, T) \leq 2^{25} \delta^{1 / 4} .
$$

We write the corollary to emphasize this is enough for the Lipschitz approximation Theorem 2.1(see proof below). But for the final goal of the $C^{\alpha}$-rugularity, the integral semi-Reifenberg condition (12) is not enough, the point-wise estimate (7) is necessary. We follow the argument as in [40, Section 24] to complete the proof.

Step 3.2' By (11) and (10), for $\delta \leq 2^{-4}, \alpha \leq 1, \forall \xi \in \operatorname{spt} \mu_{V} \cap B_{\frac{1}{2} \delta^{\frac{1}{2}} \rho}(0), \forall \sigma \leq \frac{1}{2} \delta^{\frac{1}{2}} \rho$ and $\forall x \in \operatorname{spt}_{V} \cap B_{\sigma}(\xi)$, we have

$$
\begin{aligned}
I(x): & =\int_{B_{\alpha \sigma}(\xi)}\left|p_{T_{y}}^{\perp}(x-y)\right|^{2} d \mu(y)=\int_{B_{\alpha \sigma}(\xi)} d^{2}\left(x, T_{y}\right) d \mu(y) \\
& \leq \int_{B_{(1+\alpha) \sigma}(x)} r_{x}^{2}(y)\left|\nabla^{\perp} r_{x}\right|^{2}(y) d \mu(y) \leq[(1+\alpha) \sigma]^{4} K(x) \leq 2^{11} \pi \sigma^{4} \delta^{\frac{1}{2}} .
\end{aligned}
$$

Now, take a maximal disjoint collection $\left\{B_{\frac{\alpha \sigma}{4}}\left(x_{i}\right)\right\}_{i=1}^{N}$ of balls with radius $\frac{\alpha \sigma}{4}$ and centered in $s p t \mu_{V} \cap B_{\sigma}(\xi)$. Then we have

$$
s p t \mu_{V} \cap B_{\sigma}(\xi) \subset \cup_{i=1}^{N} B_{\alpha \sigma}\left(x_{i}\right)
$$

and

$$
\mu_{V}\left(B_{\frac{\alpha \sigma}{4}}\left(x_{i}\right)\right) \geq \pi\left(\frac{\alpha \sigma}{4}\right)^{2}\left(1-2 \delta^{\frac{1}{2}}\right)
$$

Moreover, we know

$$
N \leq \frac{\mu_{V}\left(B_{\sigma}(\xi)\right)}{\pi\left(\frac{\alpha \sigma}{4}\right)^{2}\left(1-2 \delta^{\frac{1}{2}}\right)} \leq 2^{5} \frac{\mu_{V}\left(B_{\sigma}(\xi)\right)}{\pi \sigma^{2} \alpha^{2}} \leq \frac{2^{8}}{\alpha^{2}}
$$


and

$$
\int_{B_{\alpha \sigma}(\xi)} \Sigma_{i=1}^{N}\left|p_{T_{y} \Sigma}^{\perp}\left(x_{i}-y\right)\right|^{2} d \mu(y) \leq \frac{2^{8}}{\alpha^{2}} \int_{B_{\alpha \sigma}(\xi)}\left|p_{T_{y}}^{\perp}\left(x_{i}-y\right)\right|^{2} d \mu(y) \leq \frac{2^{19} \pi \sigma^{4} \delta^{\frac{1}{2}}}{\alpha^{2}} .
$$

Take $\alpha=\delta^{\frac{1}{16}}$ and $\theta=2^{21} \sigma^{2} \delta^{\frac{1}{4}}$. Then we get

$$
\frac{\mu_{V}\left(B_{\alpha \sigma}(\xi) \cap\left\{\sum_{i=1}^{N}\left|p_{T_{y}}^{\perp}\left(x_{i}-y\right)\right|^{2} \geq \theta\right\}\right)}{\mu_{V}\left(B_{\alpha \sigma}(\xi)\right)} \leq \frac{\frac{2^{19} \pi \sigma^{4} \delta^{\frac{1}{2}}}{\alpha^{2} \theta}}{\left(1-2 \delta^{\frac{1}{2}}\right) \pi(\alpha \sigma)^{2}} \leq \frac{1}{2}<1 .
$$

Thus there exists a point $y_{0} \in B_{\delta \frac{1}{16} \sigma}(\xi)$ such that

$$
\sum_{i=1}^{N}\left|p_{T_{y_{0}}}^{\perp}\left(x_{i}-y_{0}\right)\right|^{2}<\theta=2^{21} \sigma^{2} \delta^{\frac{1}{4}} .
$$

That is,

$$
\sup _{1 \leq i \leq N}\left|p_{T_{y_{0}}}^{\perp}\left(x_{i}-y_{0}\right)\right| \leq 2^{11} \sigma \delta^{\frac{1}{8}} .
$$

So, for $\forall x \in \operatorname{spt} \mu_{V} \cap B_{\sigma}(\xi) \subset \cup_{i=1}^{N} B_{\alpha \sigma}\left(x_{i}\right)=\cup_{i=1}^{N} B_{\delta \frac{1}{16} \sigma}\left(x_{i}\right)$, there exists $1 \leq i \leq N$ such that $x \in B_{\delta \frac{1}{16} \sigma}\left(x_{i}\right)$. Thus

$$
\left|p_{T_{y_{0}}}^{\perp}\left(x-y_{0}\right)\right| \leq\left|p_{T_{y_{0}}}^{\perp}\left(x-x_{i}\right)\right|+\left|p_{T_{y_{0}}}^{\perp}\left(x_{i}-y_{0}\right)\right| \leq \delta^{\frac{1}{16}} \sigma+2^{11} \sigma \delta^{\frac{1}{8}} \leq 2^{12} \delta^{\frac{1}{16}} \sigma .
$$

So, if we let $T=T_{y_{0}}+\xi-y_{0}$, then $\xi \in T$ and for any $x \in \operatorname{spt}_{V} \cap B_{\sigma}(\xi)$,

$$
\sigma^{-1} d(x, T)=\sigma^{-1}\left|p_{T}^{\perp}(x-\xi)\right| \leq \sigma^{-1}\left(\left|p_{T}^{\perp}\left(x-y_{0}\right)\right|+\left|y_{0}-\xi\right|\right) \leq 2^{13} \delta^{\frac{1}{16}} .
$$

\subsection{Lipschitz Approximation.}

Firstly, we need the following version of weighted monotonicity inequality, which roughly means most of the measure concentrate in the neighborhood of a plane.

Lemma 2.10. Assume $V=\underline{v}(\Sigma, \theta)$ is a rectifiable 2-varifold in $U$ with generalized mean curvature $H \in L^{2}\left(\mu_{V}\right)$. Then for $l \in(0,1), \beta \in(0,1 / 4), B_{R}(\xi) \subset U$ and $\forall y \in B_{\beta R}(\xi)$, we have

$$
\begin{aligned}
\pi \Theta\left(\mu_{V}, y\right) \leq & (1+24 \beta) \frac{\mu_{V}\left(\left\{x:\left|q_{0}(x-y)\right|<2 l \beta R\right\} \cap B_{R}(\xi)\right)}{R^{2}} \\
& +\frac{6}{(l \beta)^{5}} \frac{1}{R^{2}} \int_{B_{R}(\xi)}\left\|p_{T_{x} \Sigma}-p_{\mathbb{R}^{2} \times\{0\}}\right\|^{2}+\frac{2}{(l \beta)^{3}} \int_{B_{R}(\xi)}|H|^{2},
\end{aligned}
$$

where $q_{0}$ is the orthogonal projection of $\mathbb{R}^{2+k}$ onto $\{0\} \times \mathbb{R}^{k}$.

Proof. W.l.o.g., assume $\xi=0$ and $y \in B_{\beta R}(0)$. Denote $T_{y}=\mathbb{R}^{2} \times\{0\}+y$ and define $q_{y}: \mathbb{R}^{2+k} \rightarrow T_{y}^{\perp}, q_{y}\left(y+\left(x_{1}, x_{2}\right)\right)=y+x_{2}, p_{y}=i d-q_{y}$ to be the orthogonal projection to $T_{y}^{\perp}$ and $T_{y}$ respectively. For $\alpha$ to be determined, choose a function $g \in C^{1}(\mathbb{R},[0,1])$ such that $g(t) \equiv 1$ for $t \in[-\alpha R, \alpha R], g(t) \equiv 0$ for $|t| \geq 2 \alpha R$ and $\left|g^{\prime}(t)\right| \leq \frac{2}{\alpha R}$. Put $h(x)=g\left(\left|q_{y}(x)-y\right|\right)$. We will deduce a monotonicity formula involving the weight $h^{2}$. Take $X=h^{2} \eta r \nabla r\left(\right.$ here $r(x)=r_{y}(x)=|x-y|, \eta=\eta(r)$ to be determined below). Since

$$
\begin{aligned}
\operatorname{div}^{\Sigma} X & =\left\langle\nabla^{\Sigma}\left(h^{2} \eta\right), r \nabla r\right\rangle+h^{2} \eta d i v^{\Sigma}(r \nabla r) \\
& =h^{2} r \eta^{\prime}+2 h^{2} \eta+2 \eta h r\left\langle\nabla^{\Sigma} r, \nabla^{\Sigma} h\right\rangle-h^{2} r \eta^{\prime}\left|\nabla^{\perp} r\right|^{2},
\end{aligned}
$$


by the definition of generalized mean curvature $\int d i v^{\Sigma} X=-\int X \cdot H$, we know $L H:=\int h^{2}\left(r \eta^{\prime}+2 \eta\right)=\int h^{2} r \eta^{\prime}\left|\nabla^{\perp} r\right|^{2}-2 \eta h r\left\langle\nabla^{\Sigma} r, \nabla^{\Sigma} h\right\rangle-h^{2} \eta r\left\langle\nabla^{\perp} r, H\right\rangle=: R H$.

As before, since $B_{(1-\beta) R}(y) \subset B_{R}(0)$, for $0<\sigma<\rho<(1-\beta) R$, we can take

$$
\eta(r)= \begin{cases}f(\sigma)-f(\rho), & r \leq \sigma, \\ f(r)-f(\rho), & r \in(\sigma, \rho) .\end{cases}
$$

for decreasing $f$ to be chosen. Then

$$
\begin{gathered}
\eta^{\prime}(r)= \begin{cases}0, & r \leq \sigma, \\
f^{\prime}(r), & r \in(\sigma, \rho),\end{cases} \\
L H=\int_{B_{\rho} \backslash B_{\sigma}(y)} h^{2} r f^{\prime}(r)+2 \int_{B_{\sigma}(y)} h^{2}(f(\sigma)-f(\rho))+2 \int_{B_{\rho} \backslash B_{\sigma}(y)} h^{2}(f(r)-f(\rho)) \\
=\int_{B_{\rho} \backslash B_{\sigma}(y)} h^{2}\left(r f^{\prime}+2 f\right)+2 f(\sigma) \int_{B_{\sigma}(y)} h^{2}-2 f(\rho) \int_{B_{\rho}(y)} h^{2}
\end{gathered}
$$

and $R H=-2 T+R H_{1}$ for $T=\int_{B_{\rho}(y)} \eta h r\left\langle\nabla^{\Sigma} r, \nabla^{\Sigma} h\right\rangle$ and

$$
\begin{aligned}
R H_{1}= & \int_{B_{\rho} \backslash B_{\sigma}(y)} h^{2}\left\{r f^{\prime}\left|\nabla^{\perp} r\right|^{2}-r f\left\langle\nabla^{\perp} r, H\right\rangle\right\}+f(\rho) \int_{B_{\rho} \backslash B_{\sigma}(y)} h^{2} r\left\langle\nabla^{\perp} r, H\right\rangle \\
& -(f(\sigma)-f(\rho)) \int_{B_{\sigma}(y)} h^{2} r\left\langle\nabla^{\perp}, H\right\rangle \\
= & -\int_{B_{\rho} \backslash B_{\sigma}(y)} h^{2}\left|\sqrt{-r f^{\prime}} \nabla^{\perp} r+\frac{r f H}{2 \sqrt{-r f^{\prime}}}\right|^{2}+\int_{B_{\rho} \backslash B_{\sigma}(y)} h^{2}\left|\frac{r f H}{2 \sqrt{-r f^{\prime}}}\right|^{2} \\
& +f(\rho) \int_{B_{\rho}(y)} h^{2} r\left\langle\nabla^{\perp} r, H\right\rangle-f(\sigma) \int_{B_{\sigma}(y)} h^{2} r\left\langle\nabla^{\perp} r, H\right\rangle .
\end{aligned}
$$

Especially, if we take $f(r)=\frac{1}{r^{2}}$, then $r f^{\prime}+2 f=0, \sqrt{-r f^{\prime}}=\sqrt{2} r^{-1}, r f=r^{-1}$ and $\frac{r f}{2 \sqrt{-r f^{\prime}}}=\frac{1}{2 \sqrt{2}}$, then by $L H=R H$ and $-\int_{B_{\rho} \backslash B_{\sigma}(y)}\left|\frac{\nabla^{\perp} r}{r}+\frac{H}{4}\right|^{2} h^{2} \leq 0$, we get

$$
\frac{1}{\sigma^{2}} \int_{B_{\sigma}(y)} h^{2}-\frac{1}{\rho^{2}} \int_{B_{\rho}(y)} h^{2} \leq-T+T_{4}-T_{5}+\frac{1}{16} \int_{B_{\rho} \backslash B_{\sigma}(y)}|H|^{2} h^{2},
$$

where

$$
T_{4}=\frac{1}{2 \rho^{2}} \int_{B_{\rho}(y)} h^{2} r\left\langle\nabla^{\perp} r, H\right\rangle, \quad T_{5}=\frac{1}{2 \sigma^{2}} \int_{B_{\sigma}(y)} h^{2} r\left\langle\nabla^{\perp} r, H\right\rangle
$$

and

$$
\begin{aligned}
T & =\int_{B_{\rho} \backslash B_{\sigma}(y)}(f(r)-f(\rho)) h\left\langle r \nabla^{\Sigma} r, \nabla^{\Sigma} h\right\rangle+\int_{B_{\sigma}(y)}(f(\sigma)-f(\rho)) h\left\langle r \nabla^{\Sigma} r, \nabla^{\Sigma} h\right\rangle \\
& =\int_{B_{\rho} \backslash B_{\sigma}(y)} h\left\langle\frac{\nabla^{\Sigma} r}{r}, \nabla^{\Sigma} h\right\rangle+\frac{1}{\sigma^{2}} \int_{B_{\sigma}(y)} h\left\langle r \nabla^{\Sigma} r, \nabla^{\Sigma} h\right\rangle-\frac{1}{\rho^{2}} \int_{B_{\rho}(y)} h\left\langle r \nabla^{\Sigma} r, \nabla^{\Sigma} h\right\rangle \\
& =: T_{1}+T_{2}+T_{3} .
\end{aligned}
$$


By Young's inequality, we know

$$
\begin{array}{ll}
\left|T_{2}\right| \leq \frac{\varepsilon}{\sigma^{2}} \int_{B_{\sigma}(y)} h^{2}+\frac{1}{4 \varepsilon} \int_{B_{\sigma}(y)}\left|\nabla^{\Sigma} h\right|^{2}, & \left|T_{3}\right| \leq \frac{\varepsilon}{\rho^{2}} \int_{B_{\rho}(y)} h^{2}+\frac{1}{4 \varepsilon} \int_{B_{\rho}(y)}\left|\nabla^{\Sigma} h\right|^{2}, \\
\left|T_{4}\right| \leq \frac{\varepsilon}{\rho^{2}} \int_{B_{\rho}(y)} h^{2}+\frac{1}{8 \varepsilon} \int_{B_{\rho}(y)}|H|^{2} h^{2}, & \left|T_{5}\right| \leq \frac{\varepsilon}{\sigma^{2}} \int_{B_{\sigma}(y)} h^{2}+\frac{1}{8 \varepsilon} \int_{B_{\sigma}(y)}|H|^{2} h^{2},
\end{array}
$$

and

$$
\left|T_{1}\right| \leq \int_{B_{\rho} \backslash B_{\sigma}(y)} \frac{\varepsilon h^{2}}{r^{2}}+\frac{\left|\nabla^{\Sigma} h\right|^{2}}{4 \varepsilon} \leq \varepsilon \frac{\rho^{2}}{\sigma^{2}} \frac{1}{\rho^{2}} \int_{B_{\rho}(y)} h^{2}+\frac{1}{4 \varepsilon} \int_{B_{\rho} \backslash B_{\sigma}(y)}\left|\nabla^{\Sigma} h\right|^{2} .
$$

Substitute the estimate of $T_{i}, i=1,2,3,4,5$ into (13). We get, for $\varepsilon<\frac{1}{2}$,

$$
\begin{aligned}
(1-2 \varepsilon) \frac{1}{\sigma^{2}} \int_{B_{\sigma}(y)} h^{2} \leq & \left(1+2 \varepsilon+\varepsilon\left(\frac{\rho}{\sigma}\right)^{2}\right) \frac{1}{\rho^{2}} \int_{B_{\rho}(y)} h^{2} \\
& +\frac{3}{4 \varepsilon} \int_{B_{\rho}(y)}\left|\nabla^{\Sigma} h\right|^{2}+\left(\frac{1}{16}+\frac{1}{4 \varepsilon}\right) \int_{B_{\rho}(y)}|H|^{2} h^{2} .
\end{aligned}
$$

On the one hand, by definition, we know for $x \in B_{\alpha R}(y),\left|q_{y}(x)-y\right|=\left|q_{0}(x-y)\right| \leq$ $\alpha R$. Hence $h \equiv 1$ on $B_{\alpha R}(y)$. So if we take $\sigma=\alpha R<\rho<(1-\beta) R$ and use the monotonicity inequality (2), then

$$
\begin{aligned}
\pi \Theta\left(\mu_{V}, y\right) & \leq(1+\varepsilon) \frac{\mu_{V}\left(B_{\alpha R}(y)\right)}{(\alpha R)^{2}}+\frac{1}{2 \varepsilon} \int_{B_{\alpha R}(y)}|H|^{2} \\
& \leq(1+\varepsilon) \frac{1}{\sigma^{2}} \int_{B_{\sigma}(y)} h^{2}+\frac{1}{2 \varepsilon} \int_{B_{\rho}(y)}|H|^{2} \\
& \leq \frac{1+\varepsilon}{1-2 \varepsilon}\left\{\left(1+2 \varepsilon+\varepsilon\left(\frac{\rho}{\sigma}\right)^{2}\right) \frac{1}{\rho^{2}} \int_{B_{\rho}(y)} h^{2}+\frac{1}{\varepsilon} \int_{B_{\rho}(y)}\left(\left|\nabla^{\Sigma} h\right|^{2}+|H|^{2}\right)\right\},
\end{aligned}
$$

where we use $h \leq 1$ in the last inequality.

On the other hand, since $\nabla^{\mathbb{R}^{2+k}}\left|q_{y}(x)-y\right|=\frac{q_{0}(x-y)}{\left|q_{0}(x-y)\right|}$, we know for $x \in s p t \mu_{V}$ where $T_{x} \Sigma$ exists,

$$
\begin{aligned}
\left|\nabla^{\Sigma} h\right|^{2}(x) & \leq\left(\left|g^{\prime}\left(q_{0}(x-y)\right)\right|\left|\nabla^{\Sigma}\right| q_{0}(x-y)||\right)^{2} \\
& \leq\left(\frac{2}{\alpha R}\right)^{2}\left|\frac{p_{T_{x} \Sigma}\left(q_{0}(x-y)\right)}{\left|q_{0}(x-y)\right|}\right|^{2} \\
& \leq \frac{4}{(\alpha R)^{2}}\left\|p_{T_{x} \Sigma}-p_{\mathbb{R}^{2} \times\{0\}}\right\|^{2} .
\end{aligned}
$$

Moreover, sptq $\subset[-2 \alpha R, 2 \alpha R]$ implies spth $\subset\left\{x:\left|q_{0}(x-y)\right|<2 \alpha R\right\}$. Thus if we take $\rho=(1-2 \beta) R$, then $\frac{\rho}{\sigma}=\frac{1-2 \beta}{\alpha}, B_{\rho}(y) \subset B_{R}(\xi)$ and

$$
\begin{aligned}
\pi \Theta\left(\mu_{V}, y\right) \leq & \frac{1+\varepsilon}{1-2 \varepsilon}\left\{\left(1+2 \varepsilon+\varepsilon\left(\frac{\rho}{\sigma}\right)^{2}\right) \frac{1}{\rho^{2}} \int_{B_{\rho}(y)} h^{2}+\frac{1}{\varepsilon} \int_{B_{\rho}(y)}\left(\left|\nabla^{\Sigma} h\right|^{2}+|H|^{2}\right)\right\} \\
\leq & \frac{1+\varepsilon}{1-2 \varepsilon}\left\{\left(1+2 \varepsilon+\frac{(1-2 \beta)^{2} \varepsilon}{\alpha^{2}}\right) \frac{\mu_{V}\left(\left\{x:\left|q_{0}(x-y)\right|<2 \alpha R\right\} \cap B_{R}(\xi)\right)}{((1-2 \beta) R)^{2}}\right. \\
& \left.\quad+\frac{4}{\varepsilon(\alpha R)^{2}} \int_{B_{(1-2 \beta) R}(y)}\left\|p_{T_{x} \Sigma}-p_{\mathbb{R}^{2} \times\{0\}}\right\|^{2}+\frac{1}{\varepsilon} \int_{B_{(1-2 \beta) R}(y)}|H|^{2}\right\} .
\end{aligned}
$$


Take $\alpha=l \beta$ and $\varepsilon=(l \beta)^{3}=\alpha^{3} \leq 2^{-6}$ for $l<1$. Then

Thus

$$
\frac{1+\varepsilon}{1-2 \varepsilon} \leq 1+6(l \beta)^{3} \quad \text { and } \quad 1+2 \varepsilon+\frac{(1-2 \beta)^{2} \varepsilon}{\alpha^{2}} \leq 1+2 l \beta .
$$

$$
\begin{aligned}
\pi \Theta\left(\mu_{V}, y\right) \leq & \left(1+6(l \beta)^{3}\right)(1+2 l \beta) \frac{\mu_{V}\left(\left\{x:\left|q_{0}(x-y)\right|<2 l \beta R\right\} \cap B_{(1-2 \beta) R}(y)\right)}{((1-2 \beta) R)^{2}} \\
& +\frac{6}{(l \beta)^{5}} \frac{1}{R^{2}} \int_{B_{(1-2 \beta) R}(y)}\left\|p_{T_{x} \Sigma}-p_{\mathbb{R}^{2} \times\{0\}}\right\|^{2}+\frac{2}{(l \beta)^{3}} \int_{B_{(1-2 \beta) R}(y)}|H|^{2} \\
\leq & (1+24 \beta) \frac{\mu_{V}\left(\left\{x:\left|q_{0}(x-y)\right|<2 l \beta R\right\} \cap B_{R}(0)\right)}{R^{2}} \\
& +\frac{6}{(l \beta)^{5}} E(0, R, T)+\frac{2}{(l \beta)^{3}} \int_{B_{R}(0)}|H|^{2} .
\end{aligned}
$$

Corollary 2.11. Assume $\alpha, l \in(0,1)$ and $V=\underline{v}(\Sigma, \theta)$ is a rectifiable 2-varifold in $U$ with generalized mean curvature $H \in L^{2}\left(\mu_{V}\right)$ and $\frac{\mu_{V}\left(B_{R}(\xi)\right)}{\pi R^{2}} \leq 2-\alpha$. For $\beta_{1}=\beta_{1}(\alpha)=\frac{\alpha}{48(2-\alpha)}$ and $\delta=\delta(\alpha)=\frac{\alpha^{3}}{2^{23}}$, if

$$
l^{-5} E(\xi, R, T) \leq \delta^{2} \pi \text { and } l^{-3} \int_{B_{R}(\xi)}|H|^{2} \leq \delta^{2} \pi,
$$

then for $\forall y, z \in B_{\beta_{1} R}(\xi)$ with $|y-z| \geq \beta_{1} R$, we have

$$
\left|q_{0}(y-z)\right| \leq l|y-z|
$$

Proof. We assume $T=\mathbb{R}^{2} \times\{0\}$ and argue by contradiction. Otherwise, there exist $y, z \in \operatorname{spt}_{V} \cap B_{\beta_{1} R}(\xi)$ with $|y-z| \geq \beta_{1} R$ but $\left|q_{0}(y-z)\right|>l|y-z|$. Thus for $\forall x \in B_{R}(\xi)$,

$$
\left|q_{0}(x-y)\right|+\left|q_{0}(x-z)\right| \geq\left|q_{0}(y-z)\right|>l|y-z| \geq l \beta_{1} R
$$

So, either $\left|q_{0}(x-y)\right|>\frac{l \beta_{1} R}{2}$ or $\left|q_{0}(x-z)\right|>\frac{l \beta_{1} R}{2}$, i.e.,

$$
\left\{x \in B_{R}(\xi):\left|q_{0}(x-y)\right| \leq \frac{l \beta_{1} R}{2}\right\} \cap\left\{x \in B_{R}(\xi):\left|q_{0}(x-z)\right| \leq \frac{l \beta_{1} R}{2}\right\}=\emptyset .
$$

Noting $y, z \in \operatorname{spt} \mu_{V} \cap B_{\beta_{1} R}(\xi)$, by Lemma 2.10, we get

$$
\begin{aligned}
2 \pi & \leq\left(\Theta\left(\mu_{V}, y\right)+\Theta\left(\mu_{V}, z\right)\right) \pi \\
& \leq\left(1+24 \beta_{1}\right) \frac{\mu_{V}\left(\left\{x \in B_{R}(\xi):\left|q_{0}(x-z)\right|<\frac{1}{2} l \beta_{1} R \text { or }\left|q_{0}(x-z)\right|<\frac{1}{2} l \beta_{1} R\right\}\right)}{R^{2}} \\
& \quad+\frac{12}{\left(\frac{1}{2} l \beta_{1}\right)^{5}} \frac{1}{R^{2}} \int_{B_{R}(\xi)}\left\|p_{T_{x} \Sigma}-p_{\mathbb{R}^{2} \times\{0\}}\right\|^{2}+\frac{4}{\left(\frac{1}{2} l \beta_{1}\right)^{3}} \int_{B_{R}(\xi)}|H|^{2} \\
& \leq\left(1+24 \beta_{1}\right) \frac{\mu_{V}\left(B_{R}(\xi)\right)}{R^{2}}+\frac{12}{\left(\frac{1}{2} l \beta_{1}\right)^{5}} E\left(\xi, R, \mathbb{R}^{2} \times\{0\}\right)+\frac{4}{\left(\frac{1}{2} l \beta_{1}\right)^{3}} \int_{B_{R}(\xi)}|H|^{2} \\
& \leq\left(1+24 \beta_{1}\right)(2-\alpha) \pi+\frac{3 \cdot 2^{7}}{l^{5} \beta_{1}^{5}} l^{5} \delta^{2} \pi+\frac{2^{5}}{l^{3} \beta_{1}^{3}} l^{3} \delta^{2} \pi \\
& \leq\left(2-\frac{\alpha}{8}\right) \pi .
\end{aligned}
$$

A contradiction! 
Proposition 2.12 (Lipschitz Approximation 0.5 version). For $\forall \alpha \in(0,1)$, there exists

$$
\begin{aligned}
& \beta_{3}(\alpha)=\frac{1}{4} \beta_{2}(\alpha)=\frac{\alpha}{2^{8} \cdot 3 \cdot 5(4-\alpha)}, \quad \beta_{4}(\alpha)=\frac{\alpha^{3}}{2^{21}} \\
& \delta_{3}^{2}(\alpha)=\delta_{2}^{4} \delta_{1}^{10}=\frac{\alpha^{12}}{2^{174} k^{5}}\left(\delta_{1}=\frac{1}{2^{7} k}, \delta_{2}(\alpha)=\frac{\alpha^{3}}{2^{26}}\right)
\end{aligned}
$$

such that the following statement holds:

Assume $V=\underline{v}(\Sigma, \theta)$ is a rectifiable 2-varifold in $U \subset \mathbb{R}^{2+k}$ satisfying

$$
\begin{aligned}
& \text { (1) } 0 \in \operatorname{spt}_{V}, B_{R}(0) \subset U \\
& \text { (2) } \frac{\mu_{V}\left(B_{R}(0)\right)}{\pi R^{2}} \leq 2-\alpha \\
& \text { (3) } \theta \geq 1, \mu_{V}-\text { a.e. } x \in U .
\end{aligned}
$$

For any $l \in(0,1)$, if

$$
l^{-5} E:=l^{-5} E\left(0, R, \mathbb{R}^{2} \times\{0\}\right) \leq \delta_{3}^{2} \text { and } l^{-3} W:=l^{-3} \int_{B_{R}(0)} H^{2} \leq \delta_{3}^{2},
$$

then for any $\beta \in\left(\beta_{4}, \beta_{3}\right)$, there exists a Lipschitz function $f=\left(f^{1}, f^{2}, \ldots, f^{k}\right)$ : $B_{\beta R}^{\mathbb{R}^{2} \times\{0\}}(0) \rightarrow \mathbb{R}^{k}$ with

$$
\operatorname{Lipf} \leq l, \sup _{x \in B_{\beta R}}|f| \leq l \beta R
$$

and for $F=$ Graphf,

$$
\mathcal{H}^{2}\left(\left(F \backslash s p t \mu_{V}\right) \cap B_{\beta R}(0)\right)+\mu_{V}\left(B_{\beta R}(0) \backslash F\right) \leq 2^{27}\left(l^{-\frac{5}{2}} E^{\frac{1}{2}}+l^{-\frac{3}{2}} W^{\frac{1}{2}}\right) \pi R^{2} .
$$

Moreover, for the orthogonal projection $q_{0}: \mathbb{R}^{2+k} \rightarrow\{0\} \times \mathbb{R}^{k}$, we have

$$
\sup _{x \in B_{\beta R}(0) \cap s p t \mu_{V}}\left|q_{0}(x)\right| \leq l \beta R .
$$

Proof. Following the notation of Corollary 2.11, take

$$
\begin{array}{cl}
\delta_{0}(\alpha)=\beta_{0}(\alpha)=\frac{\alpha}{40}, & \delta_{1} \in(0,1) \text { to be determined } \\
\beta_{2}(\alpha)=\frac{1}{20} \beta_{1}\left(\frac{\alpha}{2}\right)=\frac{\alpha}{960(4-\alpha)}, & \delta_{2}(\alpha)=\delta\left(\frac{\alpha}{2}\right)=\frac{\alpha^{3}}{2^{26}}, \quad \delta_{3}^{2}=\delta_{2}^{4} \delta_{1}^{10} .
\end{array}
$$

The same as the proof of (8) and (9), by the monotonicity formula, it is easy to show that for $x \in B_{\beta_{0} R}(\xi) \cap \operatorname{spt} \mu_{V}$ and $\sigma \in\left(0,\left(1-\beta_{0}\right) R\right)$, we have

$$
1-2 \delta_{0} \leq \frac{\mu_{V}\left(B_{\sigma}(x)\right)}{\pi \sigma^{2}} \leq 2-\frac{\alpha}{2} .
$$

Letting

$$
G:=\left\{x \in \operatorname{spt} \mu_{V} \cap B_{\beta R}(0): \frac{E\left(x, \sigma, \mathbb{R}^{2} \times\{0\}\right)}{\left(l \delta_{1}\right)^{5}}+\frac{\int_{B_{\sigma}(x)}|H|^{2}}{\left(l \delta_{1}\right)^{3}} \leq \pi \delta_{2}^{2}, \forall \sigma<\frac{R}{10}\right\},
$$

then for any $\beta \in\left(\beta_{4}, \beta_{2}\right), x \in G$ and $y \in \operatorname{spt} \mu_{V} \cap B_{\beta R}(0)$, we have

$$
\sigma:=\frac{|x-y|}{\beta_{1}\left(\frac{\alpha}{2}\right)}<\frac{2 \beta_{2} R}{\beta_{1}\left(\frac{\alpha}{2}\right)} \leq \frac{R}{10} \leq\left(1-\beta_{0}\right) R \quad \text { and } \quad x \in B_{\beta R}(0) \subset B_{\beta_{0} R}(0) .
$$


By (14) we get

$$
\frac{\mu_{V}\left(B_{\frac{|x-y|}{\beta_{1}\left(\frac{\alpha}{2}\right)}}(x)\right)}{\pi\left(\frac{|x-y|}{\beta_{1}\left(\frac{\alpha}{2}\right)}\right)^{2}} \leq 2-\frac{\alpha}{2} .
$$

Since $x \in G$, we know that for $\sigma=\frac{|x-y|}{\beta_{1}\left(\frac{\alpha}{2}\right)}$,

$$
\frac{E\left(x, \sigma, \mathbb{R}^{2} \times\{0\}\right)}{\left(l \delta_{1}\right)^{5}}+\frac{\int_{B_{\sigma}(x)}|H|^{2}}{\left(l \delta_{1}\right)^{3}} \leq \pi \delta^{2}\left(\frac{\alpha}{2}\right) .
$$

Thus by Corollary 2.11 we know

$$
\left|q_{0}(x-y)\right| \leq l \delta_{1}|x-y| .
$$

Especially, if we take $y=0 \in \operatorname{spt}_{V} \cap B_{\beta R}(0)$, then

$$
\sup _{x \in G}\left|q_{0}(x)\right| \leq \delta_{1} l|x| \leq \delta_{1} l \beta R \text {. }
$$

Moreover, if we define $p_{0}: \mathbb{R}^{2+k} \rightarrow \mathbb{R}^{2}, p_{0}\left(x_{1}, x_{2}\right)=x_{1}$ and $\Omega_{0}=p_{0}(G)$, then for $x, y \in G$, by (15) we know

$$
\left|q_{0}(x-y)\right| \leq \frac{\delta_{1} l}{\sqrt{1-\left(\delta_{1} l\right)^{2}}}\left|p_{0}(x-y)\right| \leq \frac{2 \delta_{1} l}{\sqrt{3}}\left|p_{0}(x-y)\right|\left(\text { for } \delta_{1} \leq \frac{1}{2}\right) .
$$

Thus

$$
G=G r a p h f_{0}, \quad f_{0}\left(p_{0}(x)\right)=q_{0}(x): \Omega_{0} \rightarrow \mathbb{R}^{k}, \quad \operatorname{Lipf} f_{0} \leq \frac{2 \delta_{1} l}{\sqrt{3}} .
$$

Now by the extension theorem of Lipschitz function, there exists a Lipschitz function $\tilde{f}: \mathbb{R}^{2} \rightarrow \mathbb{R}^{k}$ such that $\tilde{f}=f$ on $\Omega_{0}$ and Lip $\tilde{f} \leq k L i p f_{0} \leq \frac{2 k \delta_{1} l}{\sqrt{3}}$. Noting that $\left|f_{0}\right| \leq \sup _{G}\left|q_{0}\right| \leq \delta_{1} l \beta R$, we can put $f=\min \{\max \{f,-l \beta R\}, l \beta R\}$ and get an extending $f$ of $f_{0}$ with

$$
\text { Lipf } \leq \frac{2 k \delta_{1} l}{\sqrt{3}} \quad \text { and } \quad \sup |f| \leq l \beta R .
$$

Noting spt $\mu_{V} \backslash G \operatorname{raph} f \subset \operatorname{spt} \mu_{V} \backslash G$, we estimate $\mu_{V}\left(\left(\operatorname{spt} \mu_{V} \backslash G\right) \cap B_{\beta R}(0)\right)$ next. For any $x \in\left(\operatorname{spt} \mu_{V} \backslash G\right) \cap B_{\beta R}(0)$, there is $\sigma_{x} \in\left(0, \frac{R}{10}\right)$ such that

$$
\left(l \delta_{1}\right)^{-5} E\left(x, \sigma_{x}, \mathbb{R}^{2} \times\{0\}\right)+\left(l \delta_{1}\right)^{-3} \int_{B_{\sigma_{x}}(x)}|H|^{2} \geq \pi \delta_{2}^{2} .
$$

Let

$$
\begin{aligned}
& A:=\left\{x \in \operatorname{spt}_{V} \cap B_{\beta R}(0):\left(l \delta_{1}\right)^{-5} E\left(x, \sigma_{x}, \mathbb{R}^{2} \times\{0\}\right) \geq \frac{1}{2} \pi \delta_{2}^{2}\right\}, \\
& B:=\left\{x \in \operatorname{spt} \mu_{V} \cap B_{\beta R}(0):\left(l \delta_{1}\right)^{-3} \int_{B_{\sigma_{x}}(x)}|H|^{2} \geq \frac{1}{2} \pi \delta_{2}^{2}\right\} .
\end{aligned}
$$

Then $\left(\operatorname{spt} \mu_{V} \backslash G\right) \cap B_{\beta R}(0) \subset A \cup B$.

By 5 -times lemma, there exists disjoint collection $\left\{B_{\sigma_{x_{j}}}\left(x_{j}\right)\right\}_{j=1}^{\infty}$ of $\left\{B_{\sigma_{x}}(x)\right\}_{x \in A}$ such that $A \subset \cup_{j=1}^{\infty} B_{5 \sigma_{x_{j}}}\left(x_{j}\right)$. For $x \in A$, we know

$$
\sigma_{x}^{2} \leq \frac{2}{\delta_{2}^{2}\left(\delta_{1} l\right)^{5}} \int_{B_{\sigma_{x}}(x)}\left\|_{T_{x} \Sigma}-p_{\mathbb{R}^{2} \times\{0\}}\right\|^{2} d \mu_{V}(x) .
$$


Since $5 \sigma_{x_{j}} \leq \frac{5 R}{10} \leq\left(1-\beta_{0}\right) R$, by (14) we know $\frac{\mu_{V}\left(B_{5 \sigma_{x_{j}}}\left(x_{j}\right)\right)}{\pi\left(5 \sigma_{x_{j}}\right)^{2}} \leq 2-\frac{\alpha}{2}$. Thus

$$
\begin{aligned}
\mu(A) & \leq \Sigma_{j=1}^{\infty} \mu\left(B_{5 \sigma_{x_{j}}}\left(x_{j}\right)\right) \leq \Sigma_{j=1}^{\infty}\left(2-\frac{\alpha}{2}\right) 25 \pi \sigma_{x_{j}}^{2} \\
& \leq 50 \pi \Sigma_{j=1}^{\infty} \frac{2}{\delta_{2}^{2}\left(\delta_{1} l\right)^{5}} \int_{B_{\sigma_{x_{j}}}\left(x_{j}\right)}\left\|p_{T_{x} \Sigma}-p_{\mathbb{R}^{2} \times\{0\}}\right\|^{2} d \mu \\
& \leq \frac{100 \pi}{\delta_{2}^{2}\left(\delta_{1} l\right)^{5}} \int_{B_{R}(0)}\left\|p_{T_{x} \Sigma}-p_{\mathbb{R}^{2} \times\{0\}}\right\|^{2} d \mu \\
& \leq 100 \pi l^{-\frac{5}{2}} R^{2} E^{\frac{1}{2}}\left(0, R, \mathbb{R}^{2} \times\{0\}\right),
\end{aligned}
$$

where we use the condition $l^{-5} E\left(0, R, \mathbb{R}^{2} \times\{0\}\right) \leq \delta_{2}^{4} \delta_{1}^{10}$ in the last inequality. Similarly, there exists disjoint collection $\left\{B_{\sigma_{y_{j}}}\left(y_{j}\right)\right\}_{j=1}^{N}$ of $\left\{B_{\sigma_{y}}(y)\right\}_{y \in B}$ such that $B \subset \cup_{j=1}^{N} B_{5 \sigma_{y_{j}}}\left(y_{j}\right)$. Since $\int_{B_{\sigma_{y}}(y)}|H|^{2} \geq \frac{1}{2} \pi \delta_{2}^{2}\left(l \delta_{1}\right)^{3}$ for any $y \in B$, we know $N \leq \frac{\int_{B_{R}(0)}|H|^{2}}{\delta_{2}^{2}\left(l \delta_{1}\right)^{3}}$ and

$$
\begin{aligned}
\mu(B) & \leq \Sigma_{j=1}^{N} \mu\left(B_{5 \sigma_{y_{j}}}\left(y_{j}\right)\right) \leq \Sigma_{j=1}^{N}\left(2-\frac{\alpha}{2}\right) 25 \pi \sigma_{y_{j}}^{2} \\
& \leq \frac{\pi}{2} N R^{2} \leq \frac{\pi R^{2}}{2 \delta_{2}^{2}\left(\delta_{1} l\right)^{3}} \int_{B_{R}(0)}|H|^{2} \\
& \leq \pi l^{-\frac{3}{2}} R^{2}\left(\int_{B_{R}(0)}|H|^{2}\right)^{\frac{1}{2}},
\end{aligned}
$$

where we use $l^{-3} \int_{B_{R}(0)}|H|^{2} \leq \delta_{2}^{4} \delta_{1}^{6}$ in the last line. As a result,

$$
\begin{aligned}
\mu_{V}\left(\left(\operatorname{spt} \mu_{V} \backslash G\right) \cap B_{\beta R}(0)\right) & \leq \pi R^{2} l^{-\frac{5}{2}}\left[100 E^{\frac{1}{2}}\left(0, R, \mathbb{R}^{2} \times\{0\}\right)+l\left(\int_{B_{R}(0)}|H|^{2}\right)^{\frac{1}{2}}\right] \\
& \leq 101 \pi R^{2} \delta_{2}^{2} \delta_{1}^{3} \leq 101 \frac{\alpha^{6}}{2^{52}} \pi R^{2} \\
\left(\text { since } \beta>\frac{\alpha^{3}}{2^{21}}\right) & <\frac{1}{2} \pi(\beta R)^{2} \\
\left(\text { by } 0 \in \operatorname{spt} \mu_{V} \text { and }(14)\right) & <\mu_{V}\left(B_{\beta R}(0)\right) .
\end{aligned}
$$

So $G \neq \emptyset$. Taking $x_{0} \in G$, by (16) and (15) we know $\left|q_{0}\left(x_{0}\right)\right| \leq \delta_{1} l \beta R$ and

$$
\sup _{y \in s p t \mu_{V} \cap B_{\beta R}(0)}\left|q_{0}(y)\right| \leq \sup _{y \in \operatorname{spt} \mu_{V} \cap B_{\beta R}(0)}\left|q_{0}\left(y-x_{0}\right)\right|+\left|q_{0}\left(x_{0}\right)\right| \leq 3 \delta_{1} l \beta R .
$$

Next, we estimate the $\mathcal{H}^{2}\left(\left(G r a p h f \backslash s p t \mu_{V}\right) \cap B_{\frac{\beta}{4} R}(0)\right)$. For this, set $F=$ Graphf and denote

$$
C:=\left(F \backslash s p t \mu_{V}\right) \cap B_{\frac{\beta}{4} R}(0) .
$$

For $\forall \eta \in C$, take $\sigma_{\eta}$ to be the smallest $\sigma$ such that $B_{\frac{\sigma}{2}}(\eta) \cap \operatorname{spt} \mu_{V}=\emptyset$ but $B_{\frac{3 \sigma}{4}}(\eta) \cap$ $s p t \mu_{V} \neq \emptyset$. Since $\eta \notin s p t \mu_{V}, \sigma_{\eta}>0$ and $0 \in \operatorname{spt} \mu_{V}$, we know $\frac{\sigma_{\eta}}{2} \leq|\eta| \leq \frac{\beta}{4} R$ and $\sigma_{\eta} \leq \frac{\beta R}{2}$. Now, $B_{\frac{3 \sigma_{\eta}}{4}}(\eta) \cap s p t \mu_{V} \neq \emptyset$ implies there is $\xi_{\eta} \in \operatorname{spt} \mu_{V} \cap B_{\frac{3 \sigma_{\eta}}{4}}(\eta) \subset$ $\operatorname{spt}_{V} \cap B_{\frac{3 \beta R}{8}}(\eta) \subset \operatorname{spt}_{V} \cap B_{\beta R}(0)$. Thus $B_{\sigma_{\eta}}(\eta) \supset B_{\frac{1}{4} \sigma_{\eta}}\left(\xi_{\eta}\right)$ and by (14) we know,

$$
\mu\left(B_{\sigma_{\eta}}(\eta)\right) \geq \mu\left(B_{\frac{1}{4} \sigma_{\eta}}\left(\xi_{\eta}\right)\right) \geq\left(1-2 \delta_{0}\right) \pi\left(\frac{1}{4} \sigma_{\eta}\right)^{2} .
$$


On the other hand, since $B \frac{\sigma_{\eta}}{2}(\eta) \cap s p t \mu=\emptyset$, by the monotonicity formula (1) we know

$$
\begin{aligned}
\int_{B_{\sigma_{\eta}}(\eta)}\left|\frac{\nabla^{\perp} r}{r}+\frac{H}{4}\right|^{2} & =\frac{\mu\left(B_{\sigma_{\eta}}(\eta)\right)}{\sigma_{\eta}^{2}}+\frac{1}{16} \int_{B_{\sigma_{\eta}}(\eta)}|H|^{2}+\frac{1}{2 \sigma_{\eta}^{2}} \int_{B_{\sigma_{\eta}}(\eta)} r\left\langle\nabla^{\perp} r, H\right\rangle \\
& \geq(1-\varepsilon) \frac{\mu\left(B_{\sigma_{\eta}}(\eta)\right)}{\sigma_{\eta}^{2}}+\left(\frac{1}{16}-\frac{1}{4 \varepsilon}\right) \int_{B_{\sigma_{\eta}}(\eta)}|H|^{2}
\end{aligned}
$$

Taking $\varepsilon=\frac{1}{2}$ in (21) and using (20), we get

$$
\begin{aligned}
\frac{\left(1-2 \delta_{0}\right) \pi \sigma_{\eta}^{2}}{16} \leq \mu\left(B_{\sigma_{\eta}}(\eta)\right) & \leq\left(2 \int_{B_{\sigma_{\eta}}(\eta)}\left|\frac{\nabla^{\perp} r}{r}+\frac{H}{4}\right|^{2}+\frac{7}{8} \int_{B_{\sigma_{\eta}}(\eta)}|H|^{2}\right) \sigma_{\eta}^{2} \\
& \leq\left(4 \int_{B_{\sigma_{\eta}}(\eta)}\left|\frac{\nabla^{\perp} r}{r}\right|^{2}+\frac{9}{8} \int_{B_{\sigma_{\eta}}(\eta)}|H|^{2}\right) \sigma_{\eta}^{2} \\
(B y(10)) & \leq\left(4 \int_{B_{\sigma_{\eta}}(\eta)}\left|\frac{p_{T_{x} \Sigma}^{\perp}(x-\eta)}{|x-\eta|^{2}}\right|^{2}+2 \int_{B_{\sigma_{\eta}}(\eta)}|H|^{2}\right) \sigma_{\eta}^{2} \\
\left(\text { Since } \operatorname{spt} \mu \cap B_{\frac{\sigma_{\eta}}{2}}(\eta)=\emptyset\right) \leq & \frac{4 \sigma_{\eta}^{2}}{\left(\frac{\sigma_{\eta}}{2}\right)^{2}} \int_{B_{\sigma_{\eta}}(\eta)}\left|p_{T_{x} \Sigma}^{\perp}\left(\frac{x-\eta}{|x-\eta|}\right)\right|^{2}+2 \sigma_{\eta}^{2} \int_{B_{\sigma_{\eta}}(\eta)}|H|^{2} \\
\left(-2 \sigma_{\eta}^{2} \int_{B_{\sigma_{\eta}}(\eta)}|H|^{2}\right) & \lesssim 32 \int_{B_{\sigma_{\eta}}(\eta)}\left\|p_{T_{x} \Sigma}^{\perp}-q_{0}\right\|^{2}+32 \int_{B_{\sigma_{\eta}}(\eta)}\left|q_{0}\left(\frac{x-\eta}{|x-\eta|}\right)\right|^{2} \\
\left(-32 \int_{B_{\sigma_{\eta}}(\eta)}\left\|p_{T_{x} \Sigma}-p_{0}\right\|^{2}\right) & \lesssim 32 \mu\left(B_{\sigma_{\eta}}(\eta) \backslash F\right)+32 \int_{B_{\sigma_{\eta}}(\eta) \cap F}\left|q_{0}\left(\frac{x-\eta}{|x-\eta|}\right)\right|^{2} \\
\left(B y \operatorname{Lipf} \leq \frac{2 k \delta_{1} l}{\sqrt{3}}\right) & \leq 32 \mu\left(B_{\sigma_{\eta}}(\eta) \backslash F\right)+32\left(\frac{2 k \delta_{1} l}{\sqrt{3}}\right)^{2} \mu\left(B_{\sigma_{\eta}}(\eta)\right) \\
& \leq 32 \mu\left(B_{\sigma_{\eta}}(\eta) \backslash F\right)+2^{6}\left(k \delta_{1} l\right)^{2}\left(2-\frac{\alpha}{2}\right) \sigma_{\eta}^{2},
\end{aligned}
$$

where we use the non-standard notation

$$
\begin{aligned}
& A_{1}+\underline{S_{1}} \\
\left(-S_{1}\right) \lesssim & A_{2}+\underline{S_{2}} \\
\left(-S_{2}\right) \lesssim & A_{3}
\end{aligned}
$$

to mean $A_{1} \leq A_{2}+S_{1} \leq A_{3}+S_{1}+S_{2}$ to save space. Fixing $\delta_{1}=\frac{1}{2^{7} k}$, then

$$
2 \sigma_{\eta}^{2} \int_{B_{\sigma_{\eta}}(\eta)}|H|^{2}+2^{6}\left(k \delta_{1} l\right)^{2}\left(2-\frac{\alpha}{2}\right) \sigma_{\eta}^{2} \leq\left(2 l^{3} \delta_{2}^{4} \delta_{1}^{6}+2^{6}\left(k \delta_{1} l\right)^{2}\left(2-\frac{\alpha}{2}\right)\right) \sigma_{\eta}^{2} \leq 2^{-6} \sigma_{\eta}^{2} .
$$

Noticing $\frac{\left(1-2 \delta_{0}\right) \pi \sigma_{\eta}^{2}}{16} \geq \frac{\sigma_{\eta}^{2}}{2^{5}}$ and substituting (23) into (22), then we get

$$
\frac{\sigma_{\eta}^{2}}{2^{5}} \leq 32\left(\int_{B_{\sigma_{\eta}}(\eta)}\left\|p_{T_{x} \Sigma}-p_{0}\right\|^{2}+\mu\left(B_{\sigma_{\eta}}(\eta) \backslash F\right)\right)+2^{-6} \sigma_{\eta}^{2},
$$

i.e.,

$$
\sigma_{\eta}^{2} \leq 2^{11}\left(\int_{B_{\sigma_{\eta}}(\eta)}\left\|p_{T_{x} \Sigma}-p_{0}\right\|^{2}+\mu\left(B_{\sigma_{\eta}}(\eta) \backslash F\right)\right)
$$


Denote $\eta^{\prime}=p_{0}(\eta)$, then the Lebesgue measure

$$
\mathcal{L}^{2}\left(B_{5 \sigma_{\eta}}^{\mathbb{R}^{2} \times\{0\}}\left(\eta^{\prime}\right)\right)=\pi\left(5 \sigma_{\eta}\right)^{2} \leq 2^{11} \cdot 5^{2} \pi\left(\int_{B_{\sigma_{\eta}}(\eta)}\left\|p_{T_{x} \Sigma}-p_{0}\right\|^{2}+\mu\left(B_{\sigma_{\eta}}(\eta) \backslash F\right)\right) .
$$

Again by the 5 -times lemma, there exists disjoint collection $\left\{B_{\sigma_{\eta_{j}}}^{\mathbb{R}^{2} \times\{0\}}\left(\eta_{j}^{\prime}\right)\right\}_{j=1}^{\infty}$ of $\left\{B_{\sigma_{\eta}}^{\mathbb{R}^{2} \times\{0\}}\left(\eta^{\prime}\right)\right\}_{\eta \in C}$ such that $p_{0}(C) \subset \cup_{j=1}^{\infty} B_{5 \sigma_{\eta_{j}}}^{\mathbb{R}^{2} \times\{0\}}\left(\eta_{j}^{\prime}\right)$. Thus

$$
\cup_{j=1}^{\infty} B_{\sigma_{\eta_{j}}}\left(\eta_{j}\right) \subset \cup_{j=1}^{\infty}\left(B_{\sigma_{\eta_{j}}}^{\mathbb{R}^{2} \times\{0\}}\left(\eta_{j}^{\prime}\right) \times \mathbb{R}^{k}\right) \cap B_{\beta R}(0) \subset B_{\beta R}(0)
$$

and

$$
\mathcal{L}^{2}\left(p_{0}(C)\right) \leq \Sigma_{j=1}^{\infty} \mathcal{L}^{2}\left(B_{5 \sigma_{\eta_{j}}}^{\mathbb{R}^{2} \times\{0\}}\left(\eta_{j}^{\prime}\right)\right) \leq 2^{18}\left(\int_{B_{\beta R}(0)}\left\|p_{T_{x} \Sigma}-p_{0}\right\|^{2}+\mu\left(B_{\beta R}(0) \backslash F\right)\right) .
$$

Moreover, since $C \subset G r a p h f$ for some $f$ with $\operatorname{Lipf} \leq 1$ and $\left(\operatorname{spt} \mu_{V} \backslash F\right) \cap B_{\beta R}$ is included in $\left(s p t \mu_{V} \backslash G\right) \cap B_{\beta R}$ whose measure has been estimated, we know

$\mathcal{H}^{2}\left((F \backslash s p t \mu) \cap B_{\frac{\beta}{4} R}(0)\right)$

$$
\begin{aligned}
& \leq(\sqrt{2})^{2} \mathcal{L}^{2}\left(p_{0}(C)\right) \\
& \leq 2^{19}\left[\int_{B_{R}(0)}\left\|p_{T_{x} \Sigma}-p_{0}\right\|^{2}+\pi R^{2} l^{-\frac{5}{2}}\left(100 E^{\frac{1}{2}}\left(0, R, \mathbb{R}^{2} \times\{0\}\right)+l\left(\int_{B_{R}(0)} H^{2}\right)^{\frac{1}{2}}\right)\right] \\
& \leq 2^{19}\left[101 \pi R^{2} l^{-\frac{5}{2}}\left(E^{\frac{1}{2}}\left(0, R, \mathbb{R}^{2} \times\{0\}\right)+l\left(\int_{B_{R}(0)} H^{2}\right)^{\frac{1}{2}}\right)\right] \\
& \leq 2^{26} \pi R^{2}\left(l^{-\frac{5}{2}} E^{\frac{1}{2}}\left(0, R, \mathbb{R}^{2} \times\{0\}\right)+l^{-\frac{3}{2}}\left(\int_{B_{R}(0)} H^{2}\right)^{\frac{1}{2}}\right)
\end{aligned}
$$

As a result, if we take $\beta_{3}(\alpha)=\frac{1}{4} \beta_{2}(\alpha)$, then for $\beta \in\left(\beta_{4}, \beta_{3}\right)$,by(17) (18)(24) and (19), we are done.

Combing this theorem with the tilt-excess estimate( Corollary 2.9), we can finish the proof of Theorem 2.1.

proof of Theorem 2.1. By (8) and Corollary 2.9 we know, for any $\xi \in \operatorname{spt} \mu_{V} \cap B_{R}(\xi)$ and $R<\frac{1}{2} \delta^{\frac{1}{2}} \rho$, there exists a plane $T=T(\xi, R)$ such that

$$
\frac{\mu\left(B_{R}(\xi)\right)}{\pi R^{2}} \leq 1+36 \delta^{\frac{1}{2}}=: 2-\alpha, \quad \text { and } \quad E(\xi, R, T) \leq 2^{25} \delta^{\frac{1}{4}} .
$$

Since $\alpha=1-36 \delta^{\frac{1}{2}} \in\left[\frac{1}{2}, 1\right]$ (for $\delta \leq \frac{1}{2^{16}}$ ), we know $\beta_{4}(\alpha) \leq \frac{1}{2^{21}} \leq \frac{1}{2^{14}} \leq \beta_{3}(\alpha)$ and $\delta_{3}^{2} \geq \frac{1}{2^{186} k^{5}}$. So if

$$
l^{-20} \delta \leq \delta_{5}:=\frac{1}{2^{844} k^{20}},
$$

then

$$
l^{-5} E(\xi, R, T) \leq \delta_{3}^{2} \text { and } l^{-3} W \leq \delta_{3}^{2}\left(\text { by } \int_{B_{R}(\xi)}|H|^{2} \leq \delta\right) .
$$

Thus by Proposition 2.12 we know, for any $\beta \in\left(\frac{1}{2^{21}}, \frac{1}{2^{14}}\right)$, there exists a Lipschitz function $f=\left(f^{1}, f^{2}, \ldots, f^{k}\right): B_{\beta R}^{T}(\xi) \rightarrow T^{\perp}$ with

$$
\operatorname{Lipf} \leq l, \sup _{x \in B_{\beta R}(\xi)}|f| \leq l \beta R
$$


and for $F=G r a p h f$,

$$
\begin{aligned}
\mathcal{H}^{2}\left(\left(F \backslash s p t \mu_{V}\right) \cap B_{\beta R}(\xi)\right) & +\mu_{V}\left(B_{\beta R}(\xi) \backslash F\right) \\
& \leq 2^{27}\left(l^{-\frac{5}{2}} E^{\frac{1}{2}}+l^{-\frac{3}{2}} W^{\frac{1}{2}}\right) \pi R^{2} \\
& \leq 2^{27}\left(l^{-\frac{5}{2}} 2^{13} \delta^{\frac{1}{8}}+l^{-\frac{3}{2}} \delta^{\frac{1}{2}}\right) \pi R^{2} \\
& \leq 2^{83} l^{-\frac{5}{2}} \delta^{\frac{1}{8}} \pi(\beta R)^{2} .
\end{aligned}
$$

Moreover, for $q: \mathbb{R}^{2+k} \rightarrow T^{\perp}$ the orthogonal projection, we have

$$
\sup _{x \in B_{\beta R}(\xi) \cap s p t \mu_{V}}|q(x)| \leq l \beta R .
$$

Especially, for $\delta \leq \delta_{6}=\delta_{5}^{2}=\frac{1}{2^{1688} k^{40}}$, we can take $l=\delta^{\frac{1}{40}}$ such that $(25)$ holds. So, if we fix $\beta=\frac{1}{2^{15}}$ and denote $\sigma=\beta R$. Then we actually proved: for $\forall \xi \in B_{\frac{\delta}{216} \rho}(0)$ and $\forall \sigma \in\left(0, \frac{\delta^{\frac{1}{2}}}{2^{16}} \rho\right)$, there exist a plane $T=T(\xi, \sigma)$ and a vector valued Lipschitz function $f=\left(f^{1}, f^{2}, \ldots, f^{k}\right): B_{\sigma}^{T}(\xi) \rightarrow T^{\perp}$ with

$$
\operatorname{Lipf} \leq \delta^{\frac{1}{40}}, \sup _{x \in B_{\sigma}(\xi)}|f| \leq \delta^{\frac{1}{40}} \sigma
$$

and for $F=G r a p h f$,

$$
\mathcal{H}^{2}\left(\left(F \backslash s p t \mu_{V}\right) \cap B_{\sigma}(\xi)\right)+\mu_{V}\left(B_{\sigma}(\xi) \backslash F\right) \leq 2^{83} \delta^{\frac{1}{16}} \pi \sigma^{2} .
$$

Moreover, for $q: \mathbb{R}^{2+k} \rightarrow T^{\perp}$ the orthogonal projection, we have

$$
\sup _{x \in B_{\sigma}(\xi) \cap s p t \mu_{V}}|q(x)| \leq \delta^{\frac{1}{40}} \sigma .
$$

\section{3. $C^{\alpha}$-Regularity}

In this section, we combine the Lipschitz approximation theorem and Reifenberg's topological theorem to finish the proof of the $C^{\alpha}$-regularity Theorem. We have proved half of it in (7). As it is noted in the last section, to show the Lipschitz approximation Theorem 2.1, the integral semi-Reifenberg condition(Corollary 2.9) is enough. We will show that Theorem 2.1 can feed back to provide another half of the Reifenberg condition. They together complete the proof of the $C^{\alpha}$-regularity.

Theorem 3.1 (Allard - Reifenberg Type Regularity). Assume $V=\underline{v}(\Sigma, \theta)$ is a rectifiable 2-varifold in $U \supset B_{\rho}(0) \subset \mathbb{R}^{2+k}$ with $0 \in \operatorname{spt} V$ and $\theta \geq 1 \mu$-a.e.x $\in$ $U$ for $\mu:=\mu_{V}:=\mathcal{H}^{2}\left\llcorner\theta\right.$. Then there exists small $\delta_{6}^{\prime}\left(=\frac{1}{2^{3536} k^{80}}\right)$ such that for any $\delta \leq \delta_{6}^{\prime}$ if

$$
\frac{\mu\left(B_{\rho}(0)\right)}{\pi \rho^{2}} \leq 1+\delta \text { and } \int_{B_{\rho}(0)}|H|^{2} \leq \delta,
$$

then for any $\xi \in B_{\frac{1}{2} \delta^{\frac{1}{2}}}(0)$ and $\sigma \in\left(0, \frac{\delta^{\frac{1}{2}}}{2^{18}} \rho\right)$, there exists a plane $T=T(\xi, \sigma)$ passing through $\xi$ such that

$$
\sigma^{-1} d_{\mathcal{H}}\left(s p t \mu_{V} \cap B_{\sigma}(\xi), T \cap B_{\sigma}(\xi)\right) \leq 2^{44} \delta^{\frac{1}{80}},
$$

Where $d_{\mathcal{H}}$ is the Hausdorff distance in $\mathbb{R}^{2+k}$. 
Moreover, for any $\alpha \in(0,1)$ and $\varepsilon=\varepsilon(k, \alpha)$ the small constant in Reifenberg's topological disk Theorem 2.6, if $2^{44} \delta^{\frac{1}{80}} \leq \varepsilon$, then spt $\mu_{V} \cap B_{\frac{1}{2^{19}} \delta^{\frac{1}{2}} \rho}(0)$ is $C^{\alpha}$ homeomorphic to a 2-dimensional topological closed disk.

Proof. For $\xi \in B_{\frac{1}{2} \delta^{\frac{1}{2}} \rho}(0)$ and $\sigma<{\frac{1}{2^{18}}}^{\frac{1}{2} \rho}$, consider the ball $B_{4 \sigma}(\xi)$. By Lemma 2.8, there exists a plane $T$ passing through $\xi$ such that for any $x \in \operatorname{spt} \mu_{V} \cap B_{4 \sigma}(\xi)$,

$$
\sigma^{-1} d(x, T) \leq 2^{15} \delta^{\frac{1}{16}} .
$$

For the same $T$, by Lemma 2.7 we know

$$
E(\xi, 2 \sigma, T) \leq 4 \int_{B_{4 \sigma}(\xi)}|H|^{2}+592 \cdot(4 \sigma)^{-2} \int_{B_{4 \sigma}(\xi)}\left(\frac{d(x, T)}{4 \sigma}\right)^{2} \leq 2^{37} \delta^{\frac{1}{8}} .
$$

Replace Corollary 2.9 by (29) in the proof of Theorem 2.1, we know that for $\delta_{5}^{\prime}=$ $\frac{1}{2^{1768} k^{40}}, l=\delta^{\frac{1}{80}}, \delta \leq \delta_{6}^{\prime}:=\delta_{5}^{\prime 2}, \beta=\frac{1}{2^{15}}$ and $2 \sigma=\beta R$, there exists a Lipschitz function $f=\left(f^{1}, f^{2}, \ldots f^{k}\right): B_{2 \sigma}(\xi) \cap T \rightarrow \mathbb{R}^{k}:=T^{\perp}$ with

$$
\text { Lipf } \leq \delta^{\frac{1}{80}}, \sup _{x^{\prime} \in B_{2 \sigma}(\xi) \cap T}\left|f\left(x^{\prime}\right)\right| \leq \delta^{\frac{1}{80}} \cdot 2 \sigma
$$

and

$$
\mathcal{H}^{2}\left((\text { graph } \backslash \text { spt } V) \cap B_{2 \sigma}(\xi)\right)+\mu_{V}\left(B_{2 \sigma}(\xi) \backslash \text { graphf }\right) \leq 2^{76} \delta^{\frac{1}{32}} \pi(2 \sigma)^{2} .
$$

Now, for any $x^{\prime} \in B_{\sigma}(\xi) \cap T$, denote $x=\left(x^{\prime}, f\left(x^{\prime}\right)\right)$ and define $d(x)=\min \left\{d\left(x, \operatorname{spt} \mu_{V} \cap\right.\right.$ $\left.\left.B_{\sigma}(\xi)\right), \frac{1}{2} \sigma\right\}$. Then for any $y^{\prime} \in B_{\frac{d(x)}{4}}\left(x^{\prime}\right) \cap B_{\left(1-2 \delta^{\frac{1}{80}}\right) \sigma}(\xi) \cap T$ and $y=\left(y^{\prime}, f\left(y^{\prime}\right)\right)$, we have

$$
d(y, x) \leq \sqrt{1+(\operatorname{Lipf})^{2}} d\left(x^{\prime}, y^{\prime}\right) \leq \sqrt{1+\delta^{\frac{1}{40}}} \frac{d(x)}{4} \leq \frac{d(x)}{2}
$$

and

$$
d(y, \xi) \leq\left|y^{\prime}-\xi\right|+\left|f\left(y^{\prime}\right)\right| \leq\left(1-2 \delta^{\frac{1}{80}}\right) \sigma+\delta^{\frac{1}{80}}(2 \sigma)=\sigma .
$$

Thus

$$
B_{\frac{d(x)}{4}}\left(x^{\prime}\right) \cap B_{\left(1-2 \delta^{\frac{1}{80}}\right) \sigma}(\xi) \cap T \subset p\left(B_{\frac{d(x)}{2}}(x) \cap B_{\sigma}(\xi)\right),
$$

where $p: \mathbb{R}^{2+k} \rightarrow T$ is the orthogonal projection. We now claim

$$
d(x) \leq 2^{43} \delta^{\frac{1}{80}} \sigma .
$$

To see this, we assume $d(x) \geq 16 \delta^{\frac{1}{80}} \sigma$ without loss of generality.

In the case $d\left(x^{\prime}, \partial B_{\left(1-2 \delta^{\frac{1}{80}}\right) \sigma}(\xi) \cap T\right) \leq 2 \delta^{\frac{1}{80}} \sigma \leq \frac{d(x)}{8}$, there exists a point $x^{\prime \prime} \in$ $\partial B_{\left(1-2 \delta \frac{1}{80}\right) \sigma}(\xi) \cap T$ such that $B_{\frac{d(x)}{4}}\left(x^{\prime}\right) \supset B_{\frac{d(x)}{8}}\left(x^{\prime \prime}\right)$. Moreover, since $d(x) \leq \frac{\sigma}{2} \leq$ $\left(1-2 \delta^{\frac{1}{80}}\right) \sigma$, we know for $x^{\prime \prime \prime}=x^{\prime \prime}+\frac{\xi-x^{\prime \prime}}{\left|\xi-x^{\prime \prime}\right|} \frac{d(x)}{16}$, there holds

$$
B_{\frac{d(x)}{8}}\left(x^{\prime \prime}\right) \cap B_{\left(1-2 \delta \frac{1}{80}\right) \sigma}(\xi) \cap T \supset B_{\frac{d(x)}{16}}\left(x^{\prime \prime \prime}\right) \cap T .
$$

In the case $x^{\prime} \in B_{\left(1-2 \delta^{\frac{1}{80}}\right) \sigma}(\xi) \cap T$, by letting $x^{\prime \prime \prime}=x^{\prime}+\frac{\xi-x^{\prime}}{\left|\xi-x^{\prime}\right|} \frac{d(x)}{16}$ we also get

$$
\left|x^{\prime \prime \prime}-\xi\right|+\frac{d(x)}{16}=\max \left\{\left|x^{\prime}-\xi\right|, \frac{d(x)}{8}-\left|x^{\prime}-\xi\right|\right\} \leq\left(1-2 \delta^{\frac{1}{80}}\right) \sigma
$$

and

$$
B_{\frac{d(x)}{16}}\left(x^{\prime \prime \prime}\right) \cap T \subset B_{\left(1-2 \delta \frac{1}{80}\right) \sigma}(\xi) \cap B_{\frac{d(x)}{4}}\left(x^{\prime}\right) \cap T .
$$


Thus in either case, by (31) we know,

$$
\mathcal{H}^{2}\left(B_{\frac{d(x)}{2}}(x) \cap \operatorname{graph} \cap \cap B_{\sigma}(\xi)\right) \geq \int_{B_{\frac{d(x)}{16}}\left(x^{\prime \prime \prime}\right) \cap T} \sqrt{1+|\nabla f|^{2}\left(y^{\prime}\right)} d y^{\prime} \geq \frac{\pi d^{2}(x)}{2^{8}} .
$$

But by the definition of $d(x)$, we know $B_{\frac{d(x)}{2}}(x) \cap s p t \mu_{V} \cap B_{\sigma}(\xi)=\emptyset$, so by (30), we get

$$
\mathcal{H}^{2}\left(B_{\frac{d(x)}{2}}(x) \cap \operatorname{graph} f \cap B_{\sigma}(\xi)\right) \leq \mathcal{H}^{2}\left((\text { graph } \backslash \operatorname{spt} V) \cap B_{2 \sigma}(\xi)\right) \leq 2^{76} \delta^{\frac{1}{32}} \pi(2 \sigma)^{2} .
$$

Combining (32) and (33), we know $d(x) \leq 2^{43} \delta^{\frac{1}{64}} \sigma \leq 2^{43} \delta^{\frac{1}{80}} \sigma$.

Moreover, since $\delta \leq \delta_{6}^{\prime}=\frac{1}{2^{3536} k^{80}} \leq \frac{1}{2^{3520}}$, we know $d(x) \leq 2^{43} \delta \frac{1}{80} \sigma<\frac{\sigma}{2}$. Thus by the definition of $d(x)$ we know $d\left(x, s p t \mu_{V} \cap B_{\sigma}(\xi)\right)=d(x) \leq 2^{41} \delta^{\frac{1}{64}} \sigma$ and hence

$$
d\left(x^{\prime}, \operatorname{spt}_{V} \cap B_{\sigma}(\xi)\right) \leq\left|f\left(x^{\prime}\right)\right|+d\left(x, \operatorname{spt} \mu_{V}\right) \leq \delta^{\frac{1}{80}}(2 \sigma)+2^{43} \delta^{\frac{1}{80}} \sigma \leq 2^{44} \delta^{\frac{1}{80}} \sigma .
$$

Combining (28) and (34), we get

$$
\sigma^{-1} d_{\mathcal{H}}\left(s p t \mu_{V} \cap B_{\sigma}(\xi), T \cap B_{\sigma}(\xi)\right) \leq \min \left\{2^{15} \delta^{\frac{1}{16}}, 2^{44} \delta^{\frac{1}{80}}\right\}=2^{44} \delta^{\frac{1}{80}} .
$$

This is the complete Reifenberg condition (27). And the second part of Theorem 3.1 is just a restatement Reifenberg's Theorem 2.6.

Remark 3.2. We do not know whether some Lipschitz regularity hold under the same condition. See Corollary 5.2 for some positive evidence.

\section{The Density Identity and Topological Finiteness}

\subsection{The Density Formula.}

This section is the start point of this paper: we are asking what is the behavior of the inverting of a minimal surface? Our first observation is the following density formula which explains the meaning of $\Theta(\Sigma, \infty)$ in the inverting setting. It turns out the result does not depend on the minimal surface equation.

Assume $\Sigma \subset \mathbb{R}^{n}$ is an immersed surface, we denote the immersion by $f: \Sigma \rightarrow \mathbb{R}^{n}$ and simply call $f: \Sigma \rightarrow \mathbb{R}^{n}$ an immersed surface. We also abuse the notations $\Sigma$ and $f(\Sigma)$ and use $\mathcal{H}^{2}\left(B_{r}(0) \cap \Sigma\right)$ to mean the Hausdorff measure of the intersection of the extrinsic ball $B_{r}(0)$ with $f(\Sigma)$. By $d \mu_{g}$ we mean the volume form of the induced metric $g=f^{*} g_{\mathbb{R}^{n}}$.

Lemma 4.1 (Density Formula). Assume $f: \Sigma \rightarrow \mathbb{R}^{n}$ is a properly immersed surface satisfying

$$
\int_{\Sigma}|H|^{2} d \mu_{g}<+\infty
$$

and

$$
\Theta_{*}(\Sigma, \infty)=\liminf _{r \rightarrow \infty} \frac{\mathcal{H}^{2}\left(B_{r}(0) \cap \Sigma\right)}{\pi r^{2}}<+\infty
$$


Let $h: \Sigma \rightarrow \mathbb{R}^{n}$ be the inverted surface, that is, $h(x)=\frac{f(x)}{|f(x)|^{2}}, \forall x \in \Sigma$. Denote $\tilde{\Sigma}=h(\Sigma), \tilde{H}=$ the mean curvature of $\tilde{\Sigma}$ and $\tilde{g}=d h \otimes d h$. Then we have the locally antisymmetric transformation formula

$$
\left(\frac{|\tilde{H}|^{2}}{16}-\left|\frac{\tilde{H}}{4}+\frac{\tilde{\nabla} \perp \tilde{r}}{\tilde{r}}\right|^{2}\right) d \mu_{\tilde{g}}=-\left(\frac{|H|^{2}}{16}-\left|\frac{H}{4}+\frac{\nabla^{\perp} r}{r}\right|^{2}\right) d \mu_{g}
$$

for $r=|f|$ and $\tilde{r}=|h|$. Moreover, the density

$$
\Theta(\Sigma, \infty):=\lim _{r \rightarrow+\infty} \frac{\mathcal{H}^{2}\left(\Sigma \cap B_{r}(0)\right)}{\pi r^{2}}
$$

at infinity is well-defined and satisfies the global representation formula

$$
\int_{\tilde{\Sigma} \backslash\{0\}}\left(\frac{|\tilde{H}|^{2}}{16}-\left|\frac{\tilde{H}}{4}+\frac{\tilde{\nabla}^{\perp} \tilde{r}}{\tilde{r}}\right|^{2}\right) d \mu_{\tilde{g}}= \begin{cases}\pi \Theta(\Sigma, \infty) & 0 \notin \Sigma, \\ \pi(\Theta(\Sigma, \infty)-\Theta(\Sigma, 0)) & 0 \in \Sigma,\end{cases}
$$

where $\Theta(\Sigma, 0)=\lim _{r \rightarrow 0} \frac{\mu_{g}\left(\Sigma \cap B_{r}(0)\right)}{\pi r^{2}}$.

Proof. Denote $\tilde{g}=\left\langle\frac{\partial h}{\partial x^{i}}, \frac{\partial h}{\partial x^{j}}\right\rangle d x^{i} \otimes d x^{j}$. Then,

$$
\begin{gathered}
h_{i}=|f|^{-2} f_{i}-2 f|f|^{-4}\left\langle f, f_{i}\right\rangle, \\
h_{i j}=|f|^{-2} f_{i j}-2|f|^{-4}\left\langle f, f_{j}\right\rangle f_{i}-2|f|^{-4}\left\langle f, f_{i}\right\rangle f_{j}+8|f|^{-6} f\left\langle f, f_{i}\right\rangle\left\langle f, f_{j}\right\rangle \\
-2|f|^{-4} f\left\langle f, f_{i j}\right\rangle-2|f|^{-4} f\left\langle f_{i}, f_{j}\right\rangle,
\end{gathered}
$$

and

$$
\tilde{g}_{i j}=\left\langle h_{i}, h_{j}\right\rangle=|f|^{-4} g_{i j}, \quad \tilde{g}=|f|^{-4} g \quad \text { and } \quad \tilde{g}^{i j}=|f|^{4} g^{i j} .
$$

So

$$
\tilde{H}=\tilde{g}^{i j}\left(h_{i j}-\left\langle h_{i j}, h_{k}\right\rangle \tilde{g}^{k l} h_{l}\right)=|f|^{4} g^{i j} h_{i j}-|f|^{8}\left\langle g^{i j} h_{i j}, h_{k}\right\rangle g^{k l} h_{l},
$$

where

$$
\begin{aligned}
g^{i j} h_{i j}= & |f|^{-2} g^{i j} f_{i j}-4|f|^{-4} g^{i j}\left\langle f, f_{j}\right\rangle f_{i}+8|f|^{-6}\left|f^{\top}\right|^{2} f \\
& -2|f|^{-4}\left\langle f, g^{i j} f_{i j}\right\rangle-4|f|^{-4} f
\end{aligned}
$$

and

$$
\begin{aligned}
\left\langle g^{i j} h_{i j}, h_{k}\right\rangle= & \left(|f|^{-2} g^{i j} f_{i j}-4|f|^{-4} g^{i j}\left\langle f, f_{j}\right\rangle f_{i}+8|f|^{-6}\left|f^{\top}\right|^{2} f\right. \\
& \left.-2|f|^{-4}\left\langle f, g^{i j} f_{i j}\right\rangle-4|f|^{-4} f\right) \cdot\left(|f|^{-2} f_{k}-2|f|^{4}\left\langle f, f_{k}\right\rangle f\right) \\
= & |f|^{-4}\left\langle g^{i j} f_{i j}, f_{k}\right\rangle .
\end{aligned}
$$

Thus

$$
\begin{aligned}
\tilde{H}= & |f|^{4}\left(|f|^{-2} g^{i j} f_{i j}-4|f|^{-4} g^{i j}\left\langle f, f_{j}\right\rangle f_{i}+8|f|^{-6}\left|f^{\top}\right|^{2} f-2|f|^{-4} f\left\langle f, g^{i j} f_{i j}\right\rangle\right. \\
& \left.-4|f|^{-4} f\right)-|f|^{4}\left\langle g^{i j} f_{i j}, f_{k}\right\rangle g^{k l}\left(|f|^{-2} f_{l}-2|f|^{-4}\left\langle f, f_{l}\right\rangle f\right) \\
= & |f|^{2} H-4 f^{\top}+8|f|^{-2}\left|f^{\top}\right|^{2} f-2 f\left\langle f, g^{i j} f_{i j}\right\rangle-4 f+2\left\langle g^{i j} f_{i j}, f^{\top}\right\rangle f \\
= & |f|^{2} H-4 f^{\top}+8|f|^{-2}\left|f^{\top}\right|^{2} f-2 f\langle H, f\rangle-4 f
\end{aligned}
$$

where in the last step we use the equation $\left\langle g^{i j} f_{i j}, f-f^{\top}\right\rangle=\left\langle\left(g^{i j} f_{i j}\right)^{\perp}, f\right\rangle=\langle H, f\rangle$. 
Since $h=\frac{f}{|f|^{2}}$, we know $|h|^{2}=\frac{1}{|f|^{2}}, \tilde{g}^{i j}=|f|^{4} g^{i j}=\frac{1}{|h|^{4}} g^{i j}$ and $f=\frac{h}{|h|^{2}}$, $f_{i}=|h|^{-2} h_{i}-2|h|^{-4}\left\langle h, h_{i}\right\rangle h, f^{\perp}=f-g^{i j}\left\langle f, f_{i}\right\rangle f_{j}$. By

$$
\left\langle f, f_{i}\right\rangle=\left\langle\frac{h}{|h|^{2}}, \frac{1}{|h|^{2}} h_{i}-\frac{2}{|h|^{4}}\left\langle h, h_{i}\right\rangle h\right\rangle=\frac{\left\langle h, h_{i}\right\rangle}{|h|^{4}}-\frac{2\left\langle h, h_{i}\right\rangle}{|h|^{4}}=-\frac{\left\langle h, h_{i}\right\rangle}{|h|^{4}},
$$

we have

$$
f^{\perp}=\frac{h}{|h|^{2}}+|h|^{4} \tilde{g}^{i j} \frac{\left\langle h, h_{i}\right\rangle}{|h|^{4}}\left(\frac{h_{j}}{|h|^{2}}-\frac{2\left\langle h, h_{j}\right\rangle h}{|h|^{4}}\right)=\frac{h^{\top}}{|h|^{2}}+\frac{|h|^{2}-2\left|h^{\top}\right|^{2}}{|h|^{4}} h .
$$

Thus

$$
\begin{gathered}
\left|f^{\perp}\right|^{2}=\frac{\left|h^{\top}\right|^{2}}{|h|^{4}}+\frac{2\left(|h|^{2}-2\left|h^{\top}\right|^{2}\right)}{|h|^{6}}\left|h^{\top}\right|^{2}+\frac{\left(|h|^{2}-2\left|h^{\top}\right|^{2}\right)^{2}}{|h|^{6}}=\frac{\left|h^{\perp}\right|^{2}}{|h|^{4}}, \\
f^{\top}=f-f^{\perp}=-\frac{h^{\top}}{|h|^{2}}+\frac{2\left|h^{\top}\right|^{2} h}{|h|^{4}}, \quad\left|f^{\top}\right|^{2}=|f|^{2}-\left|f^{\perp}\right|^{2}=\frac{\left|h^{\top}\right|^{2}}{|h|^{4}},
\end{gathered}
$$

and

$$
\begin{aligned}
\tilde{H} & =|f|^{2} H-2\langle H, f\rangle f-4 f^{\top}+\frac{8\left|f^{\top}\right|^{2}}{|f|^{2}} f-4 f \\
& =\frac{H}{|h|^{2}}-\frac{2}{|h|^{4}}\langle H, h\rangle h+4\left(\frac{|h|^{2} h^{\top}-2\left|h^{\top}\right|{ }^{2} h}{|h|^{4}}\right)+\frac{8 \frac{\left|h^{\top}\right|^{2}}{|h|^{4}}}{\frac{|h|^{2}}{|h|^{4}}} \frac{h}{|h|^{2}}-4 \frac{h}{|h|^{2}} \\
& =\frac{H}{|h|^{2}}-\frac{2}{|h|^{4}}\langle H, h\rangle h-4 \frac{h^{\perp}}{|h|^{2}} .
\end{aligned}
$$

Moreover, for $\tilde{r}=|h|$, we know $\tilde{\nabla}^{\perp} \tilde{r}=\frac{h^{\perp}}{|h|}$. So, by (40) and (41) we get,

$$
\left\langle\tilde{H}, \frac{h^{\perp}}{|h|^{2}}\right\rangle=\left\langle\frac{H}{|h|^{2}}-\frac{2}{|h|^{4}}\langle H, h\rangle h-4 \frac{h^{\perp}}{|h|^{2}}, \frac{h}{|h|^{2}}\right\rangle=-|f|^{2}\langle H, f\rangle-4\left|f^{\perp}\right|^{2}
$$

and

$$
\begin{aligned}
-\left(\frac{\left.\tilde{H}\right|^{2}}{16}-\left|\frac{\tilde{H}}{4}+\frac{\tilde{\nabla} \perp}{\tilde{r}}\right|^{2}\right) d \mu_{\tilde{g}} & =\left(\frac{1}{2}\left\langle\tilde{H}, \frac{h^{\perp}}{|h|^{2}}\right\rangle+\left|\frac{h^{\perp}}{|h|^{2}}\right|^{2}\right)|f|^{-4} d \mu_{g} \\
& =\left(-\frac{1}{2}|f|^{2}\langle H, f\rangle-2\left|f^{\perp}\right|^{2}+\left|f^{\perp}\right|^{2}\right)|f|^{-4} d \mu_{g} \\
& =-\left(\frac{\left|f^{\perp}\right|^{2}}{|f|^{4}}+\frac{1}{2}\left\langle H, \frac{f^{\perp}}{|f|^{2}}\right\rangle\right) d \mu_{g} \\
& =\left(\frac{|H|^{2}}{16}-\left|\frac{H}{4}+\frac{\nabla^{\perp} r}{r}\right|^{2}\right) d \mu_{g} .
\end{aligned}
$$

The following argument belongs to [22, Appendix]. By (36), (35) and Corollary 2.4 , we know $\Theta^{*}(\Sigma, \infty)<+\infty$ and

$$
\int_{\Sigma}\left|\frac{\nabla^{\perp} r}{r}\right|^{2}<+\infty
$$

Thus for any $\varepsilon>0$, there exists $\rho_{0}>0$ such that for any $\rho \geq \rho_{0}$, we have

$$
\int_{\Sigma \backslash B_{\rho_{0}}}|H|^{2} d \mu_{g} \leq \varepsilon \quad \text { and } \quad \frac{\mathcal{H}^{2}\left(\Sigma \cap B_{\rho}(0)\right)}{\pi \rho^{2}} \leq \Theta^{*}(\Sigma, \infty)+\varepsilon .
$$


On the one hand,

$$
\left|\frac{1}{2 \rho^{2}} \int_{B_{\rho}(0)}\left\langle r \nabla^{\perp} r, H\right\rangle d \mu_{g}\right| \leq \frac{1}{2 \rho} \int_{B_{\rho_{0}}(0)}|H|+\frac{\pi \varepsilon^{\frac{1}{2}}}{2}\left(\Theta^{*}(\Sigma, \infty)+\varepsilon\right)^{\frac{1}{2}} .
$$

Letting $\rho \rightarrow \infty$ first and then $\varepsilon \rightarrow 0$, we get

$$
\lim _{\rho \rightarrow \infty} \frac{1}{2 \rho^{2}} \int_{B_{\rho}(0)}\left\langle r \nabla^{\perp} r, H\right\rangle d \mu_{g}=0 .
$$

On the other hand, by

$$
\left|\frac{1}{2 \sigma^{2}} \int_{B_{\sigma}(0)}\left\langle r \nabla^{\perp} r, H\right\rangle d \mu_{g}\right| \leq \frac{1}{2}\left(\frac{\mathcal{H}^{2}\left(\Sigma \cap B_{\sigma}(0)\right)}{\sigma^{2}} \int_{\Sigma \cap B_{\sigma}(0)}|H|^{2}\right)^{\frac{1}{2}},
$$

we also know

$$
\lim _{\sigma \rightarrow 0} \frac{1}{2 \sigma^{2}} \int_{B_{\sigma}(0)}\left\langle r \nabla^{\perp} r, H\right\rangle d \mu_{g}=0
$$

So, by (35)(43)(44)(45) and letting $\rho \rightarrow \infty$ and $\sigma \rightarrow 0$ in the monotonicity formula (1), we know $\Theta(\Sigma, \infty)$ is well-defined and satisfies

$$
\begin{aligned}
\pi(\Theta(\Sigma, \infty)-\Theta(\Sigma, 0)) & =-\int_{\Sigma}\left(\frac{|H|^{2}}{16}-\left|\frac{H}{4}+\frac{\nabla^{\perp} r}{r}\right|^{2}\right) d \mu_{g} \\
& =\int_{\tilde{\Sigma} \backslash\{0\}}\left(\frac{|\tilde{H}|^{2}}{16}-\left|\frac{\tilde{H}}{4}+\frac{\tilde{\nabla} \perp \tilde{r}}{\tilde{r}}\right|^{2}\right) d \mu_{\tilde{g}}
\end{aligned}
$$

where in the last line we use (37).

Remark 4.2. In the special case of minimal surfaces, the density formula goes like

$$
\int_{\tilde{\Sigma} \backslash\{0\}}|\tilde{H}|^{2} d \mu_{\tilde{g}}=16 \int_{\Sigma}\left|\frac{\nabla^{\perp} r}{r}\right|^{2} d \mu_{g}= \begin{cases}16 \pi \Theta(\Sigma, \infty) & 0 \notin \Sigma, \\ 16 \pi(\Theta(\Sigma, \infty)-\Theta(\Sigma, 0)) & 0 \in \Sigma .\end{cases}
$$

It means the density of a minimal surface can dominate the Willmore energy of its inverted surface $\tilde{\Sigma}$. But in general, the inverted surface $\tilde{\Sigma}$ has singularity at the inverted point 0 and the density formula can not dominate the topology of geometry(say total curvature) of $\tilde{\Sigma}$. For example, the family of Scherk's singlyperiodic minimal surfaces have density two at infinity, but they all have infinity genuses.

Remark 4.3. As it is seen, the locally antisymmetric transformation formula (37) and then density formula follows easily from direct calculation. But how such a term occurs? Here we give an explanation in the setting of conformal deformation of submanifolds. Recall there are two conformal invariances for surfaces, the extrinsic local one

$$
\left|A-\frac{H}{n} g\right|_{g}^{2} d \mu_{g}=\left|\tilde{A}-\frac{\tilde{H}}{n} \tilde{g}\right|_{\tilde{g}}^{2} d \mu_{\tilde{g}}
$$

and the intrinsic global one- the Gauss-Bonnet formula

$$
\int_{\Sigma} K d \mu_{g}=\int_{\Sigma} \tilde{K} d \mu_{\tilde{g}}
$$


Under conformal setting, the global Gauss-Bonnet formula has a local explanation. Assume $\tilde{g}=e^{2 u} g$ is a conformal metric on a closed Riemann surface $(\Sigma, g)$. Applying Stokes' formula to the Yamabe equation

$$
\triangle_{g} u-K+\tilde{K} e^{2 u}=0,
$$

we get

$$
\int_{\Sigma} K d \mu_{g}=\int_{\Sigma} \tilde{K} e^{2 u} d \mu_{g}=\int_{\Sigma} \tilde{K} d \mu_{\tilde{g}}
$$

For the same reason, in higher dimensional, assume $\tilde{g}=u^{\frac{4}{n-2}} g$ and apply the Stokes formula to the Yamabe equation

$$
\triangle_{g} u-\frac{n-2}{4(n-1)} S u+\frac{n-2}{4(n-1)} \tilde{S} u^{\frac{n+2}{n-2}}=0 .
$$

We get

$$
\int_{M} S u d \mu_{g}=\int_{M} \tilde{S} \tilde{u} d \mu_{\tilde{g}}
$$

where $\tilde{u}=u^{-1}$ satisfies $g=\tilde{u}^{\frac{4}{n-2}} \tilde{g}$. Note both sides contain the conformal factors $(u, \tilde{u})$. So, in high dimension, the invariance is not in a conformal class, but just for a conformal pair $(g, \tilde{g})$. With this experience, we guess the corresponding extrinsic invariant should also admit the shape of

$$
\star u d \mu_{g}=\tilde{\star} \tilde{u} d \mu_{\tilde{g}}(\text { local })
$$

or

$$
\int_{M} \star u d \mu_{g}=\int_{M} \tilde{\star} \tilde{u} d \mu_{\tilde{g}} \text { (global) . }
$$

For example, for high dimensional analogue of (47), we assume $M^{n} \subset N^{n+k}$ and $(G, \tilde{G})$ are a pair of conformal metrics on $N^{n+k}$ with conformal factors $(U, \tilde{U})$, i.e., $\tilde{G}=U^{\frac{4}{n-2}} G$ (note the index $n=\operatorname{dim} M$ ) and $\tilde{U}=U^{-1}$. Denote $u=\left.U\right|_{M}, \tilde{u}=\left.\tilde{U}\right|_{M}$ and assume $(g, \tilde{g})$ are the induced metrics of $M \subset(N,(G, \tilde{G}))$. Then $\tilde{g}=u^{\frac{4}{n-2}} g$ and direct calculus shows high dimensional analogue of (47) is of type (49):

$$
\left|A-\frac{H}{n} g\right|_{g}^{2} u d \mu_{g}=\left|\tilde{A}-\frac{\tilde{H}}{n} \tilde{g}\right|_{\tilde{g}}^{2} \tilde{u} d \mu_{\tilde{g}} .
$$

This is a local one. A natural question is, what extrinsic global invariance is corresponding to the intrinsic global invariance (48). We take $n \geq 3$ as an example. For this, we take the trace of the restriction of Ricci tensor of $G$ on $M$, i.e., denote

$$
S_{g}^{G}=\operatorname{tr}_{g} \operatorname{Ric}(N, G)
$$

and call it the extrinsic scalar curvature. The goal is to find the invariance of type (50) involving $R_{g}^{G}$. As in the intrinsic case, the first step is to calculate the equation of the extrinsic scalar curvature when the background metric deforms conformally. The result is

$$
d i v^{M} \nabla U+\frac{n}{n-2} \frac{\left|\nabla^{\perp} U\right|^{2}}{u}-\frac{n-2}{4(n-1)} S_{g}^{G} u+\frac{n-2}{4(n-1)} S_{\tilde{g}}^{\tilde{G}} u^{\frac{n+2}{n-2}}=0,
$$

wherediv ${ }^{M} \nabla U$ means the extrinsic divergence of the restriction of the gradient of $U$ on $M$ and $\nabla^{\perp} U$ represent the projection of $\nabla U$ to the normal bundle $T^{\perp} M$. Since (51) reduces to the Yamabe equation when $M=N$, we call it extrinsic Yamabe 
equation. The next is to apply the Stokes formula to the extrinsic Yamabe equation. Note the extrinsic divergence theorem goes like

$$
\int_{M} d i v^{M} \nabla U d \mu_{g}=-\int_{M} \nabla U \cdot H d \mu_{g},
$$

where $H$ is the mean curvature of the submanifold $(M, g) \subset(N, G)$. We get the global equation

$$
\begin{aligned}
\tilde{C}:=\int_{M} S_{\tilde{g}}^{\tilde{G}} \tilde{u} d \mu_{\tilde{g}}= & \int_{M} S_{g}^{G} u d \mu_{g} \\
& +\int_{M}\left(\frac{4(n-1)}{n-2}\left\langle\frac{\nabla U}{u}, H\right\rangle-\frac{4(n-1) n}{(n-2)^{2}} \frac{\left|\nabla^{\perp} U\right|^{2}}{u^{2}}\right) u d \mu_{g} \\
= & : C+Q^{\prime} .
\end{aligned}
$$

This equation looks not so symmetrically as we expected. To make (52) to possess the symmetry of type (50), we guess the term $Q^{\prime}$ is a global antisymmetric term, i.e, $\tilde{Q}^{\prime}=-Q^{\prime}$. If so, then (52) become the symmetric form

$$
\tilde{C}+\frac{\tilde{Q}^{\prime}}{2}=C+\frac{Q^{\prime}}{2} .
$$

It turns out that $Q^{\prime}$ is not only globally antisymmetric, but also comes form a local conformal antisymmetry:

$$
\begin{aligned}
\tilde{Q} & :=\left(\frac{1}{n-2}\left\langle\frac{\tilde{\nabla} \tilde{U}}{\tilde{u}}, \tilde{H}\right\rangle_{\tilde{g}}-\frac{n}{(n-2)^{2}}\left|\frac{\tilde{\nabla}^{\perp} \tilde{U}}{\tilde{u}}\right|_{\tilde{g}}^{2}\right) \tilde{u} d \mu_{\tilde{g}} \\
& =-\left(\frac{1}{n-2}\left\langle\frac{\nabla U}{u}, H\right\rangle_{g}-\frac{n}{(n-2)^{2}}\left|\frac{\nabla^{\perp} U}{u}\right|_{g}^{2}\right) u d \mu_{g}=-Q .
\end{aligned}
$$

So (52) becomes the symmetric form of type (50), i.e.,

$$
\int_{M}\left(S_{\tilde{g}}^{\tilde{G}}+T_{\tilde{g}}^{\tilde{G}}\right) \tilde{u} d \mu_{\tilde{g}}=\int_{M}\left(S_{g}^{G}+T_{g}^{G}\right) u d \mu_{g}
$$

where, $T_{g}^{G}=2(n-1) Q=\frac{2(n-1)}{n-2}\left\langle\frac{\nabla U}{u}, H\right\rangle_{g}-\frac{2 n(n-1)}{(n-2)^{2}}\left|\frac{\nabla^{\perp} U}{u}\right|_{g}^{2}$.

The above calculation is in a compact manifold, but the antisymmetry (53) is a local form, which also holds in noncompact ambient space. Especially, when we are caring about submanifolds in $\mathbb{R}^{n+k}$ and the conformal factor is induced by the inversion, (53) coincides with the locally antisymmetric transformation formula (37) in dimension $n=2$, which is a key observation in getting the density identity.

Remark 4.4. In the case (36) does not holds, i.e., $\Theta_{*}(\Sigma, \infty)=+\infty$, its natural to define $\Theta(\Sigma, \infty)=+\infty$. So, by the lemma, for a properly immersed surface in $\mathbb{R}^{n}$ with $\int_{\Sigma}|H|^{2} d \mu_{g}<+\infty$, the density $\Theta(\Sigma, \infty)=\lim _{r \rightarrow \infty} \frac{\mathcal{H}^{2}\left(\Sigma \cap B_{r}(0)\right)}{\pi r^{2}}$ is always well-defined, whether it is finite of infinite. In this sense, Lemma 4.1 holds without the assumption of (36). Only in the case $\Theta(\Sigma, \infty)=+\infty$, by (37) and Corollary 2.4 , both side of (38) are infinite.

\subsection{The Density Identity.}

Firstly, we need the following weak(in varifold sense) removability of singularity. 
Proposition 4.5. Assume $f: \Sigma \rightarrow \mathbb{R}^{n}$ is a properly immersed surface satisfying (35) and (36) and $\tilde{\Sigma}=h(\Sigma)$ is its inverted surface. Then for any $r \in(0, \infty)$,

$$
\mu_{\tilde{g}}\left(B_{r}(0) \backslash\{0\}\right) \leq \frac{C e^{4}}{\left(e^{2}-1\right)} \pi r^{2},
$$

where $C=9 \Theta_{*}(\Sigma, \infty)+\frac{59}{16 \pi} \int_{\Sigma}|H|^{2} d \mu_{g}$. And we have

$$
\int_{\tilde{\Sigma} \backslash\{0\}}|\tilde{H}|^{2} d \mu_{\tilde{g}}<+\infty .
$$

Moreover, if we extend $\mu_{\tilde{g}}$ and $\tilde{H}$ trivially across $0 \in \mathbb{R}^{n}$, then for vector field $X \in C_{0}^{1}\left(\mathbb{R}^{n}, \mathbb{R}^{n}\right)$ (do not need to be supported in $\mathbb{R}^{n} \backslash\{0\}$ ), we have

$$
\int_{\mathbb{R}^{n}} \operatorname{div} \tilde{\Sigma}^{\tilde{\Sigma}} X d \mu_{\tilde{g}}=-\int_{\mathbb{R}^{n}}\langle X, \tilde{H}\rangle d \mu_{\tilde{g}} .
$$

That is, $\tilde{\Sigma}$ is a varifold in $\mathbb{R}^{n}$ with generalized mean curvature $\tilde{H} \in L^{2}\left(\mu_{\tilde{g}}\right)$.

Proof. By (35), (36) and (3) in Corollary 2.4, we know for any $\rho \in(0, \infty)$,

$$
\frac{\mathcal{H}^{2}\left(B_{\rho}(0) \cap \Sigma\right)}{\pi \rho^{2}} \leq C,
$$

where $C=9 \Theta_{*}(\Sigma, \infty)+\frac{59}{16 \pi} \int_{\Sigma}|H|^{2} d \mu_{g}$. Since $\tilde{g}=\frac{1}{|f|^{4}} g$, we know $d \mu_{\tilde{g}}=\frac{1}{|f|^{4}} d \mu_{g}$. So, for $r=e^{-t}>0$,

$$
\begin{aligned}
& \mu_{\tilde{g}}\left(\tilde{\Sigma} \cap B_{r} \backslash\{0\}\right)=\lim _{\varepsilon \rightarrow 0} \int_{\tilde{\Sigma} \cap\left(B_{r} \backslash B_{\varepsilon}\right)} d \mu_{\tilde{g}}=\lim _{\varepsilon \rightarrow 0} \int_{\Sigma \cap\left(B_{\frac{1}{\varepsilon}} \backslash B_{\frac{1}{r}}\right)} \frac{1}{|f|^{4}} d \mu_{g}
\end{aligned}
$$

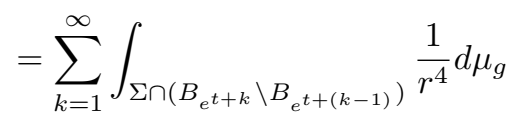

$$
\begin{aligned}
& \leq \sum_{k=1}^{\infty} \frac{C \pi e^{2(t+k)}}{e^{4(t+(k-1))}}=\frac{C \pi e^{4}}{\left(e^{2}-1\right)} r^{2} .
\end{aligned}
$$

By (39), we note

$$
\begin{aligned}
\tilde{H} & =|f|^{2} H-2\langle H, f\rangle f-4 f^{\top}+\frac{8\left|f^{\top}\right|^{2}}{|f|^{2}} f-4 f \\
& =|f|^{2} H-2\langle H, f\rangle f+4 f^{\perp}-\frac{8\left|f^{\perp}\right|^{2}}{|f|^{2}} f .
\end{aligned}
$$

Thus

$$
|\tilde{H}|^{2} \leq 320\left(|f|^{4}|H|^{2}+\left|f^{\perp}\right|^{2}\right)
$$

and

$$
\begin{aligned}
\int_{\tilde{\Sigma} \cap\left(B_{\rho}(0) \backslash\{0\}\right)}|\tilde{H}|^{2} d \mu_{\tilde{g}} & \leq 320 \int_{\Sigma \backslash B_{\frac{1}{\rho}}}\left(|f|^{4}|H|^{2}+\left|f^{\perp}\right|^{2}\right)|f|^{-4} d \mu_{g} \\
& \leq 320 \int_{\Sigma}\left(|H|^{2}+\left|\frac{\nabla^{\perp} r}{r}\right|^{2}\right) d \mu_{g} .
\end{aligned}
$$


By Corollary 2.4 again, the right hand term is finite. So, letting $\rho \rightarrow+\infty$ and we get

$$
\int_{\tilde{\Sigma} \backslash\{0\}}|\tilde{H}|^{2} d \mu_{\tilde{g}} \leq 320 \int_{\Sigma}\left(|H|^{2}+\left|\frac{\nabla^{\perp} r}{r}\right|^{2}\right)<+\infty .
$$

Finally, by (55), (57) and cut-off argument(see [22, Appendix]), we know $\tilde{\Sigma}$ is a varifold in $\mathbb{R}^{n}$ with generalized mean curvature $\tilde{H} \in L^{2}\left(\mu_{\tilde{g}}\right)$.

With this proposition, we know the monotonicity formula holds for the varifold $\tilde{\Sigma}$ with generalized mean curvature $\tilde{H}$ and can be used to show the following density identity.

Lemma 4.6 (Density identity). Assume $f: \Sigma \rightarrow \mathbb{R}^{n}$ is a properly immersed surface satisfying (35) and (36) and $\tilde{\Sigma}=h(\Sigma)$ is its inverted surface. If the base point $0 \notin \Sigma$, then

$$
\Theta(\tilde{\Sigma}, 0):=\lim _{\sigma \rightarrow 0} \frac{\mu_{\tilde{g}}\left(\tilde{\Sigma} \cap B_{\sigma}(0)\right)}{\sigma^{2}}=\Theta(\Sigma, \infty) \geq 1 .
$$

Proof. In this case, for $0<\sigma<\rho<\infty$, we have the monotonicity formula

$$
\begin{aligned}
\frac{\mu_{\tilde{g}}\left(\tilde{\Sigma} \cap B_{\sigma}\right)}{\sigma^{2}} & =\frac{\mu_{\tilde{g}}\left(\tilde{\Sigma} \cap B_{\rho}\right)}{\rho^{2}}+\frac{1}{2 \rho^{2}} \int_{\tilde{\Sigma} \cap B_{\rho}}\left\langle\tilde{r} \tilde{\nabla}^{\perp} \tilde{r}, \tilde{H}\right\rangle-\frac{1}{2 \sigma^{2}} \int_{\tilde{\Sigma} \cap B_{\sigma}}\left\langle\tilde{r} \tilde{\nabla}{ }^{\perp} \tilde{r}, \tilde{H}\right\rangle \\
& +\frac{1}{16} \int_{\tilde{\Sigma} \cap\left(B_{\rho} \backslash B_{\sigma}\right)}|\tilde{H}|^{2}-\int_{\tilde{\Sigma} \cap\left(B_{\rho} \backslash B_{\sigma}\right)}\left|\frac{\tilde{\nabla} \perp \tilde{r}}{\tilde{r}}+\frac{\tilde{H}}{4}\right|^{2}
\end{aligned}
$$

On the one hand, by (57) and (55), we know

$$
\lim _{\sigma \rightarrow 0} W(\sigma):=\lim _{\sigma \rightarrow 0} \int_{\tilde{\Sigma} \cap B_{\sigma}}|\tilde{H}|^{2}=0
$$

and

$$
\lim _{\sigma \rightarrow 0}\left|\frac{1}{2 \sigma^{2}} \int_{\tilde{\Sigma} \cap B_{\sigma}}\left\langle\tilde{r} \tilde{\nabla}^{\perp} \tilde{r}, \tilde{H}\right\rangle\right| \leq \lim _{\sigma \rightarrow 0} \frac{1}{2}\left(\frac{\mu_{\tilde{g}}\left(\tilde{\Sigma} \cap B_{\sigma}\right)}{\sigma^{2}}\right)^{1 / 2} W(\sigma)^{1 / 2}=0 .
$$

On the other hand, the properness of $f$ and $0 \notin \Sigma$ implies $\tilde{\Sigma} \backslash B_{\sigma}(0)$ is compact. So we have

and

$$
\lim _{\rho \rightarrow \infty} \frac{\mu_{\tilde{g}}\left(\tilde{\Sigma} \cap B_{\rho}\right)}{\rho^{2}}=0
$$

$$
\lim _{\rho \rightarrow+\infty}\left|\frac{1}{2 \rho^{2}} \int_{\tilde{\Sigma} \cap B_{\rho}}\left\langle\tilde{r} \tilde{\nabla}^{\perp} \tilde{r}, \tilde{H}\right\rangle\right| \leq \lim _{\rho \rightarrow+\infty} \frac{1}{2}\left(\frac{\mu_{\tilde{g}}\left(\tilde{\Sigma} \cap B_{\rho}\right)}{\rho^{2}}\right)^{1 / 2}\left(\int_{\tilde{\Sigma}}|\tilde{H}|^{2}\right)^{1 / 2}=0 .
$$

Letting $\rho \rightarrow \infty$ and $\sigma \rightarrow 0$ in (58) and applying the density formula (38), we get

$$
\Theta(\tilde{\Sigma}, 0)=\int_{\tilde{\Sigma} \backslash\{0\}}\left(\frac{|\tilde{H}|^{2}}{16}-\left|\frac{\tilde{H}}{4}+\frac{\tilde{\nabla} \tilde{\nabla}^{\perp}}{\tilde{r}}\right|^{2}\right) d \mu_{\tilde{g}}=\Theta(\Sigma, \infty) .
$$

Noting the inverted surface $\tilde{\Sigma}$ is smooth away from 0 , by Lemma 2.3 and the properness of $f$, we know

$$
\Theta(\Sigma, \infty)=\Theta(\tilde{\Sigma}, 0) \geq \limsup _{y \rightarrow 0} \Theta(\tilde{\Sigma}, y) \geq 1
$$




\subsection{Topological Finiteness.}

The density identity and density formula implies the single term $\Theta(\Sigma, \infty)$ can control both the Willmore energy and the local density of the inverted surface. So, when combining with the Allard-Reifenberg type $C^{\alpha}$ regularity Theorem 3.1, we can prove the main theorem.

Proposition 4.7. Assume $f: \Sigma \rightarrow \mathbb{R}^{n}$ is a properly immersed surface with finite Willmore energy. For any $R>0$, let $\Sigma_{1}$ be a noncompact connected component of $\Sigma \backslash f^{-1}\left(B_{R}(0)\right)$. Then

$$
\Theta\left(\Sigma_{1}, \infty\right):=\lim _{r \rightarrow \infty} \frac{\mathcal{H}^{2}\left(\Sigma_{1} \cap B_{r}(0)\right.}{\pi r^{2}} \geq 1 .
$$

Moreover, there exists an $\varepsilon=\varepsilon(n)>0$ such that if

$$
\Theta\left(\Sigma_{1}, \infty\right) \leq 1+\varepsilon(n)
$$

then there is an $R_{2} \geq R$ such that for any $r \geq R_{2}, \Sigma_{1} \backslash f^{-1}\left(B_{r}\right)$ is homeomorphic to $S^{1} \times \mathbb{R}$ and $f: \Sigma_{1} \backslash f^{-1}\left(B_{r}\right) \rightarrow \mathbb{R}^{n}$ is embedding.

Proof. Since $\Sigma$ is proper, we know $\Sigma_{1}$ has compact boundary, thus can be extended to be a complete surface in $\mathbb{R}^{n}$ without boundary by gluing a compact surface $\Sigma_{2}$ with $\partial \Sigma_{2}=-\partial \Sigma_{1}$. So we can assume $\Sigma_{1}$ to be a surface properly immersed in $\mathbb{R}^{n}$ without boundary and satisfies (35). Thus by Remark 4.4 and Lemma 4.6, we know $\Theta\left(\Sigma_{1}, \infty\right):=\lim _{r \rightarrow \infty} \frac{\mathcal{H}^{2}\left(\Sigma_{1} \cap B_{r}(0)\right.}{\pi r^{2}}$ is well-defined and $\Theta\left(\Sigma_{1}, \infty\right) \geq 1$.

Moreover, in the case $\Theta\left(\Sigma_{1}, \infty\right) \leq 1+\varepsilon$, choose a base point $x_{0} \notin \Sigma_{1}$, define $h(x)=\frac{f(x)-x_{0}}{\left|f(x)-x_{0}\right|^{2}}+x_{0}$ and denote the inverted surface by $\tilde{\Sigma}_{1}=h\left(\Sigma_{1}\right)$. Then by Proposition 4.5 and Lemma 4.6 , we know $\tilde{\Sigma}_{1}$ is a rectifiable 2-varifold in $\mathbb{R}^{n}$ with generalized mean curvature $\tilde{H} \in L^{2}\left(\mathbb{R}^{n}, d \mu_{\tilde{g}}\right)$ and

$$
\Theta\left(\tilde{\Sigma}_{1}, x_{0}\right)=\frac{1}{16} \int_{\tilde{\Sigma}_{1}}|\tilde{H}|^{2}-\int_{\tilde{\Sigma}_{1}}\left|\frac{\tilde{\nabla}^{\perp} \tilde{r}}{\tilde{r}}+\frac{\tilde{H}}{4}\right|^{2}=\Theta\left(\Sigma_{1}, \infty\right) \in[1,1+\varepsilon) .
$$

So, there exists $\rho_{0}>0$ such that for any $\rho<\rho_{0}$, we have

$$
\frac{\mathcal{H}^{2}\left(\tilde{\Sigma}_{1} \cap B_{\rho}\left(x_{0}\right)\right)}{\pi \rho^{2}} \leq 1+2 \varepsilon, \text { and } \int_{\tilde{\Sigma}_{1} \cap B_{\rho}\left(x_{0}\right)}|\tilde{H}|^{2}<\varepsilon
$$

Since $\tilde{\Sigma}_{1}$ is smooth outside the base point $x_{0}$, we know $\Theta(x) \geq 1$ for every $x \in \tilde{\Sigma}_{1}$. Taking $\varepsilon=\varepsilon(n)$ small enough and applying Theorem 3.1 we know $\tilde{\Sigma}_{1} \cap B_{\sigma}\left(x_{0}\right)$ is a topological disk for $\sigma \leq \frac{1}{2^{19}}(2 \varepsilon)^{\frac{1}{2}} \rho_{0}$, which implies the conclusion.

Remark 4.8. By a geometric measure theory argument of E.Kuwert, Y.X.Li and R.Schätzle (see [22, Appendix] and [21]), it is directly shown $\Theta\left(\Sigma_{1}, \infty\right) \geq 1$ and if $\Theta\left(\Sigma_{1}, \infty\right)<2$, then $\Theta\left(\Sigma_{1}, \infty\right)=1$. We sketch the proof for reader's convenience.

Proof. We also assume $\Theta\left(\Sigma_{1}, \infty\right)<+\infty$. Extend $\Sigma_{1}$ to be smooth and boundary free and still denote it by $\Sigma_{1}$. Take the current $T_{r}=\left(\frac{1}{r}\right)_{\sharp} \Sigma_{1}$ and the varifold $\mu_{r}=\left(\frac{1}{r}\right)_{\sharp}\left(\mathcal{H}^{2}\left\llcorner\Sigma_{1}\right)\right.$. By $(44)$, for any $R>0$,

$$
\lim _{r \rightarrow \infty}\left\|\delta \mu_{r}\right\|\left(B_{R}(0)\right)=\lim _{r \rightarrow \infty} \frac{\int_{\Sigma_{1} \cap B_{r R}(0)}|H| d \mu_{g}}{r}=0 .
$$

So, by the compactness of varifold [14][40, Theorem 32.2 and Lemma 26.14] and the compactness of integral varifolds[1],[40, Theorem 42.7 and Remark 42.8], there 
exist an integral current $T_{\infty}$, a stationary integral varifold $\mu_{\infty}$ and a sequence of $r_{i} \rightarrow+\infty$ such that

$$
\begin{aligned}
& T_{r_{i}} \rightarrow T_{\infty} \text { ( weak convergence as currents ), } \\
& \mu_{r_{i}} \rightarrow \mu_{\infty} \text { ( weak convergence as varifolds). }
\end{aligned}
$$

Since $\mu_{\infty}$ is a Radon measure, we know for fixed $x$ and $\mathcal{L}^{1}$-almost every $\rho>0$, $\mu_{\infty}\left(\partial B_{\rho}(x)\right)=0$ and

$$
\frac{\mu_{\infty}\left(B_{\rho}(x)\right)}{\pi \rho^{2}}=\lim _{i \rightarrow+\infty} \frac{\mathcal{H}^{2}\left(\Sigma_{1} \cap B_{r_{i}}(x)\right)}{\pi\left(r_{i} \rho\right)^{2}}=\Theta\left(\Sigma_{1}, \infty\right) .
$$

Since $\mu_{\infty}$ is stationary and integral, by the monotonicity formula and the upper semi-continuity, we know

$$
\Theta\left(\Sigma_{1}, \infty\right)=\Theta\left(\mu_{\infty}, \infty\right)=\Theta\left(\mu_{\infty}, x\right) \geq \limsup _{y \rightarrow x} \Theta\left(\mu_{\infty}, y\right) \geq 1 .
$$

Moreover, when $\Theta\left(\Sigma_{1}, \infty\right)<2$, noting $\lim _{i \rightarrow \infty}\left\|\delta \mu_{r_{i}}\right\|\left(B_{R}(0)\right)=0$ for any $R>0$ and $\Theta\left(\mu_{r_{i}}, \infty\right) \equiv \Theta\left(\Sigma_{1}, \infty\right)<2$, by the same argument as in [21, Proposition 2.2], we get

$$
\mu_{\infty}=\mu_{T_{\infty}}
$$

So $\Theta\left(\mu_{T_{\infty}}, \infty\right)=\Theta\left(\mu_{\infty}, \infty\right) \in[1,2)$ and by [21, Theorem 2.1], we know $T_{\infty}$ is a plane. Thus

$$
\Theta\left(\Sigma_{1}, \infty\right)=\Theta\left(\mu_{\infty}, 0\right)=\Theta\left(\mu_{T_{\infty}}, 0\right)=1 .
$$

As a corollary, our main theorem is a global version of the above topological rigidity Proposition 4.7.

Theorem 4.9 (Finite Topology). Assume $f: \Sigma \rightarrow \mathbb{R}^{n}$ is a properly immersed surface with finite Willmore energy. Then

$$
e(\Sigma, \infty) \leq \Theta(\Sigma, \infty) .
$$

Moreover, if we assume

$$
e(\Sigma, \infty)>\Theta(\Sigma, \infty)-1<\infty
$$

then

1) $\Sigma$ has finite topology;

2) $\Theta(\Sigma, \infty)=e(\Sigma, \infty)=$ : $e$ is an integer and $\Sigma$ has exact $e$ ends with density one.

3) $\Sigma$ has finite total curvature, i.e., $\int_{\Sigma}|A|^{2} d \mu_{g}<+\infty$;

4) $\Sigma$ is conformal to a closed Riemann surface with $e(\Sigma, \infty)$ points removed.

Proof. There is nothing to prove if $\Theta(\Sigma, \infty)=+\infty$. So we assume $\Theta(\Sigma, \infty)<$ $+\infty$. By the properness, for each $r>0, \Sigma \cap B_{r}(0)$ has the connected components decomposition $\Sigma \cap B_{r}(0)=K_{r} \sqcup \sqcup_{i \in I(r)} \Sigma_{i, r}$, where $K_{r}$ is the compact part and each $\Sigma_{i, r}$ is noncompact. By Proposition 4.7, we get for each $i \in I(r), \Theta\left(\Sigma_{i, r}, \infty\right) \geq 1$. Since these $\left\{\Sigma_{i, r}\right\}_{i \in I(r)}$ are disjoint, we know

$$
|I(r)| \leq \sum_{i \in I(r)} \Theta\left(\Sigma_{i, r}, \infty\right) \leq \Theta(\Sigma, \infty)<+\infty .
$$

Letting $r \rightarrow+\infty$, we know

$$
e(\Sigma, \infty)=\lim _{r \rightarrow \infty}|I(r)| \leq \Theta(\Sigma, \infty)
$$


Moreover, if $e(\Sigma, \infty)>\Theta(\Sigma, \infty)-1$, then there exists $r_{0}>0$ such that $e(\Sigma, \infty)=$ $\left|I\left(r_{0}\right)\right|>\Theta(\Sigma, \infty)-1$. So by $(59)$ and $\Theta\left(\Sigma_{i, r_{0}}, \infty\right) \geq 1$, we know

$$
\Theta\left(\Sigma_{i, r_{0}}, \infty\right)<2, \forall i \in I\left(r_{0}\right) .
$$

By Remark 4.8 we know in fact

$$
\Theta\left(\Sigma_{i, r_{0}}, \infty\right)=1
$$

Thus $e(\Sigma, \infty)=\Theta(\Sigma, \infty)$ and by Proposition 4.7 again, there exists $r_{1}>r_{0}$ such that for every $r \geq r_{1}$, each $\Sigma_{i, r_{0}} \backslash B_{r}(0)$ is an embedded annulus in $\mathbb{R}^{n}$. Take $r$ large enough such that $K_{r_{0}} \subset B_{r}(0)$. Then $\Sigma \backslash B_{r}(0)=\sqcup_{i \in I\left(r_{0}\right)}\left(\Sigma_{i, r_{0}} \backslash B_{r}(0)\right)$ consists of $\left|I\left(r_{0}\right)\right|=e(\Sigma, \infty)$ many properly embedded annulus. By properness, $\Sigma \cap B_{r}(0)$ is compact, so $\Sigma$ is homeomorphic to a closed surface with $e(\Sigma, \infty)$ points removed. Now, by Ilmanen's local Gauss-Bonnet estimate [18, Theorem 3], we know for each $r<s<\infty$ and $\varepsilon>0$,

$$
(1-\varepsilon) \int_{\Sigma \cap B_{r}(0)}|A|^{2} d \mu_{g} \leq \int_{\Sigma \cap B_{s}(0)}|H|^{2} d \mu_{g}+8 \pi g\left(\Sigma \cap B_{s}(0)\right)+\frac{24 \pi D^{\prime} s^{2}}{\varepsilon(s-r)^{2}},
$$

where $g\left(\Sigma \cap B_{s}(0)\right)$ is the genus of the closed surface obtained by capping off the boundary of $\Sigma \cap B_{s}(0)$ by disks and $D^{\prime}=\sup _{t \in[r, s]} \frac{\mathcal{H}^{2}\left(\Sigma \cap B_{t}(0)\right)}{\pi t^{2}}$. Since we have shown $\Sigma$ has finite topology, by letting $s \rightarrow \infty$ and then $r \rightarrow \infty$ and taking $\varepsilon=\frac{1}{2}$, we get

$$
\int_{\Sigma}|A|^{2} d \mu_{g} \leq 2 \int_{\Sigma}|H|^{2} d \mu_{g}+16 \pi g(\Sigma)+96 \pi \Theta(\Sigma, \infty)<+\infty .
$$

So, by Huber's classification[17] of complex structures for complete surfaces with finite total curvature, each end of $\Sigma$ is parabolic, i.e., $\Sigma$ is conformal to a closed Riemann surface with $e(\Sigma, \infty)$ points removed.

Remark 4.10. The surfaces in Theorem 4.9 have finite topology and finite total curvature, but it is impossible to dominate their topology or total curvature by the Willmore energy and density of such surfaces. For example, Hoffman and Meeks find[16] there are a family of embedded minimal surfaces with three multiplicity one ends but arbitrary many genuses. Their total curvature also tend to infinity as the genus goes to infinity.

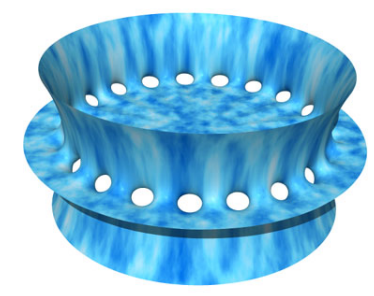

The Costa-Hoffman-Meeks surface with many handles 


\section{Applications}

\subsection{Isolated Singularities.}

In this subsection, we will care about the isolated singularity and do inverse process of section 4 .

Proposition 5.1. Assume $\Sigma \subset B_{1}(0) \backslash\{0\} \subset \mathbb{R}^{2+k}$ is a properly immersed surface with $\partial \Sigma \subset \partial B_{1}(0)$,

$$
\int_{\Sigma \backslash\{0\}}|H|^{2} d \mathcal{H}^{2}<+\infty
$$

and

$$
\Theta_{*}(\Sigma, 0)=\liminf _{r \rightarrow 0} \frac{\mathcal{H}^{2}\left(\Sigma \cap B_{r}(0)\right)}{\pi r^{2}}<+\infty .
$$

Then the inverted surface $\tilde{\Sigma}$ is properly immersed in $\mathbb{R}^{n+k}$ with finite density $\Theta(\tilde{\Sigma}, \infty) \geq 1$ at infinity and

$$
\int_{\tilde{\Sigma}}|\tilde{H}|^{2} d \mathcal{H}^{2}<+\infty
$$

Moreover, there holds the density identity

$$
\Theta(\Sigma, 0)=\Theta(\tilde{\Sigma}, \infty)
$$

which means both sides are well-defined and they are equal.

Proof. Since $\partial \Sigma \subset \partial B_{1}(0)$ is compact, we can close it up and assume $\Sigma \subset B_{2}(0)$ is a surface without boundary. By (61) and (62) and the same argument as in Proposition 4.5 , we know $\bar{\Sigma}=\Sigma \cup\{0\}$ is an integral varifold in $B_{2}(0)$ with generalized mean curvature $H \in L^{2}$. So, the monotonicity formula (1) holds and $\Theta(\Sigma, 0) \geq 1$ is well defined. Noting $\bar{\Sigma}$ has finite volume, by Corollary 2.4, we know

$$
\int_{\Sigma}\left|\frac{\nabla^{\perp} r}{r}\right|^{2} d \mathcal{H}^{2}<+\infty
$$

Hence by letting $\sigma \rightarrow 0$ and $\rho \rightarrow \infty$ in (1), we get

$$
\pi \Theta(\Sigma, 0)=\int_{\Sigma}\left(\frac{|H|^{2}}{16}-\left|\frac{H}{4}+\frac{\nabla^{\perp} r}{r}\right|^{2}\right) d \mathcal{H}^{2} .
$$

Also use $f: \Sigma \rightarrow B_{2}(0) \subset \mathbb{R}^{2+k}$ to denote the immersion map and let $h=\frac{f}{|f|^{2}}$ be the inversion. Again by the observation (56). We know for any $R>0$,

$$
\int_{\tilde{\Sigma} \cap B_{R}(0)}|\tilde{H}|^{2} d \mathcal{H}^{2} \leq 320 \int_{\Sigma \backslash B_{\frac{1}{R}(0)}}\left(|H|^{2}+\left|\frac{\nabla^{\perp} r}{r}\right|^{2}\right) d \mathcal{H}^{2} .
$$

Letting $R \rightarrow \infty$, we get $\int_{\tilde{\Sigma}}|\tilde{H}|^{2} d \mathcal{H}^{2}<+\infty$ and the monotonicity formula (58) holds for $\tilde{\Sigma}$.

Now, on the one hand, since $\tilde{\Sigma} \subset \mathbb{R}^{2+k} \backslash B_{\frac{1}{2}}(0)$, we know for $\sigma<\frac{1}{2}$,

$$
\frac{\mathcal{H}^{2}\left(\tilde{\Sigma} \cap B_{\sigma}(0)\right)}{\sigma^{2}}=\frac{1}{2 \sigma^{2}} \int_{B_{\sigma}(0)}\left\langle\tilde{r} \tilde{\nabla}{ }^{\perp} \tilde{r}, \tilde{H}\right\rangle d \mathcal{H}^{2}=0 .
$$

On the other hand, by (42), we know

$$
\left|\left\langle\tilde{\nabla}{ }^{\perp} \tilde{r}, \tilde{H}\right\rangle\right| \leq 4\left|f^{\perp}\right|^{2}+|f|^{2}\left|\left\langle H, f^{\perp}\right\rangle\right| \leq 5\left|f^{\perp}\right|^{2}+|H|^{2}|f|^{4} .
$$


So,

$$
\begin{aligned}
\left|\frac{1}{2 \rho^{2}} \int_{\tilde{\Sigma} \cap B_{\rho}(0)}\left\langle\tilde{r} \tilde{\nabla}{ }^{\perp} \tilde{r}, \tilde{H}\right\rangle d \mu_{\tilde{g}}=\right| & \leq \frac{5}{2 \rho^{2}} \int_{\Sigma \backslash B_{\frac{1}{\rho}}(0)} \frac{1}{|f|}\left(\left|f^{\perp}\right|^{2}+|f|^{4}|H|^{2}\right) \frac{1}{|f|^{4}} d \mu_{g} \\
& \leq \frac{5}{2 \rho} \int_{\Sigma}\left(|H|^{2}+\left|\frac{\nabla^{\perp} r}{r}\right|^{2}\right) d \mu_{g} .
\end{aligned}
$$

Letting $\sigma \rightarrow 0$ and $\rho \rightarrow \infty$ in (58), we get

$$
\begin{aligned}
\Theta(\tilde{\Sigma}, \infty) & =-\lim _{\rho \rightarrow \infty, \sigma \rightarrow 0} \frac{1}{\pi} \int_{\tilde{\Sigma} \cap\left(B_{\rho} \backslash B_{\sigma}\right)}\left(\frac{|\tilde{H}|^{2}}{16}-\left|\frac{\tilde{H}}{4}+\frac{\tilde{\nabla} \tilde{r}^{2}}{\tilde{r}}\right|^{2}\right) d \mu_{\tilde{g}} \\
& =\lim _{\rho \rightarrow \infty, \sigma \rightarrow 0} \frac{1}{\pi} \int_{\Sigma \cap\left(B_{\frac{1}{\sigma}} \backslash B_{\frac{1}{\rho}}\right)}\left(\frac{|H|^{2}}{16}-\left|\frac{H}{4}+\frac{\nabla^{\perp} r}{r}\right|^{2}\right) d \mu_{g} \\
& =\Theta(\Sigma, 0),
\end{aligned}
$$

where we use the local antisymmetric transformation formula (37) and (63).

Similar to the conception of the number of ends at infinity, for a surface $\Sigma$ properly immersed in $B_{1}(0) \backslash\{0\}$, we define the number of local connected components of $\Sigma$ near 0 by

$$
e(\Sigma, 0)=\lim _{r \rightarrow 0} \tilde{\beta}_{0}\left(\Sigma \cap B_{r}(0) \backslash\{0\}\right)
$$

where by $\tilde{\beta}_{0}$ we mean the number of noncompact connected components of a topology space.

Corollary 5.2. Assume $\Sigma \subset B_{1}(0) \backslash\{0\} \subset \mathbb{R}^{2+k}$ is a properly immersed surface with $\partial \Sigma \subset \partial B_{1}(0)$ and satisfying (60) and

$$
e(\Sigma, 0)>\Theta_{*}(\Sigma, 0)-1<\infty .
$$

Then $\Sigma$ has finite topology and finite total curvature. Moreover, we also know $\Theta(\Sigma, 0)=e(\Sigma, 0)$ is an integer and for small $r>0$, and $1 \leq i \leq e(\Sigma, 0)$, each component $\left(\left(\Sigma_{i} \cup\{0\}\right) \cap B_{r}(0), g\right)$ is bi-Lipschitz homeomorphic to a 2-dimensional disk.

Proof. Without loss of generality, we assume $e(\Sigma, 0)=1$ and $\Theta_{*}(\Sigma, 0)<2$. By Proposition 5.1, the inverted surface $\tilde{\Sigma}$ is properly immersed in $\mathbb{R}^{2+k}$ with finite Willmore energy and

$$
1 \leq \Theta(\tilde{\Sigma}, \infty)=\Theta(\Sigma, 0)<2 .
$$

So, by Theorem 4.9, $\tilde{\Sigma}$ has finite topology and finite total curvature, is conformal to a punctured disk when restricted to the outside of a large ball and has density $\Theta(\tilde{\Sigma}, \infty)=1$. So, $\Sigma \cap B_{r}(0)$ is conformal to a punctured disk for small $r$, i.e., there is a conformal parametrization $\varphi: D_{1}(0) \backslash\{0\} \rightarrow \Sigma \cap B_{r}(0)$. Noting the trace free part of the second fundamental form is conformal invariant, We know

$$
\begin{aligned}
\int_{\Sigma}|A|^{2} d \mu_{g} & \leq 2 \int_{\Sigma}\left|A-\frac{H}{2} g\right|^{2} d \mu_{g}+\int_{\Sigma}|H|^{2} d \mu_{g} \\
& =2 \int_{\tilde{\Sigma}}\left|\tilde{A}-\frac{\tilde{H}}{2} \tilde{g}\right|^{2}+\int_{\Sigma}|H|^{2} d \mu_{g} \\
& \leq 4 \int_{\tilde{\Sigma}}|\tilde{A}|^{2} d \mu_{\tilde{g}}+2 \int_{\tilde{\Sigma}}|\tilde{H}|^{2} d \mu_{\tilde{g}}+\int_{\Sigma}|H|^{2} d \mu_{g}<+\infty
\end{aligned}
$$


By Kuwert and Li's classification theorem[20, Theorem 3.1] for isolated singularities of surfaces with finite area and finite total curvature, we know $\varphi \in W^{2,2}\left(D, \mathbb{R}^{2+k}\right)$ and the induced conformal metric $g=e^{2 u}\left(d x^{2}+d y^{2}\right)$ satisfying

$$
u(z)=m \log z+w(z)
$$

for $w(z) \in C^{0} \cap W^{1,2}(D)$ and $m=\Theta(\Sigma, 0)-1$. Now, since $\Theta(\Sigma, 0)=\Theta(\tilde{\Sigma}, \infty)=1$, we know $m=0$. Hence $u=w \in C^{0}(D)$, which means

$$
\frac{1}{C}|x-y| \leq d_{g}(f(x), f(y))=\inf _{\gamma \text { joining } x, y} \int_{0}^{1} e^{u(\gamma)}|\dot{\gamma}| d t \leq C|x-y|,
$$

i.e., $f: D \rightarrow\left(\bar{\Sigma} \cap B_{r}(0), g\right)$ is a bi-Lipschitz parametrization for $r$ small.

Remark 5.3. The same conclusion holds for surfaces properly immersed in a punctured geodesic ball $B_{1}(p) \backslash\{p\}$ of a Riemannian manifold $\left(M^{2+k}, g\right)$, since $(M, g)$ can be embedded in $R^{2+k+N}$ by Nash embedding theorem and the density, topology and finiteness of Willmore energy of $\Sigma$ will not change.

\subsection{Uniqueness of The Catenoid and Minimal Ends.}

As a corollary, we prove a uniqueness result for the catenoid.

Corollary 5.4. Assume $\Sigma \subset \mathbb{R}^{3}$ is a connected properly immersed minimal surface with at least two ends. If

$$
\Theta(\Sigma, \infty)<3
$$

then $\Sigma$ is the catenoid.

Proof. Since $e(\Sigma, \infty) \geq 2>\Theta(\Sigma, \infty)-1$, by Theorem 4.9, we know $\Sigma$ has finite total curvature and exactly two embedded ends. So, by Schoen's uniqueness theorem[34], $\Sigma$ is a catenoid.

As mentioned in the introduction, this uniqueness of the catenoid is also a direct corollary of Leon Simon's theorem on the uniqueness of the tangent cone[41][42, The paragraph after Theorem 5.7]. The following is a most simple example of such uniqueness phenomenon.

Corollary 5.5. Assume $\Sigma$ is a complete immersed minimal surface in $\mathbb{R}^{2+k}$ with

$$
\Theta(\Sigma,+\infty)<e+1 \quad \text { and } \quad e(\Sigma, \infty) \geq e .
$$

Then $\Sigma$ has exactly e ends and each end $\Sigma_{i}$ can be written as a graph over some plane $V_{i}$ in with gradient tends to be zero. Moreover, in the case $k=1$, these $T_{i}$ are the same.

Proof. Since $\Sigma$ is complete and of quadratic area growth, by [10, Lemma 3], the immersion $f$ is proper. By Theorem 4.9, there exist $r_{1}>0$ such that

$$
\Sigma \backslash B_{r_{1}}(0)=\sqcup_{i=1}^{e} \Sigma_{i},
$$

where $e=e(\Sigma, \infty)$ and each $\Sigma_{i}$ is conformal to a punctured disk with finite total curvature and $\Theta\left(\Sigma_{i}, \infty\right)=1$. Moreover, since $\Sigma_{i}$ is minimal, its Gaussian map $G(x)=e_{1}(x) \wedge e_{2}(x): \Sigma_{i} \rightarrow\left(G_{2, n}(\mathbb{R}), g_{c}\right)$ is a harmonic map on the punctured disk with finite energy(note the energy of the Gaussian map is exactly the total curvature). So by Sacks and Uhlenbeck's [32, Theorem 3.6] removability of singularity for harmonic maps with finite energy(or [15, Theorem A]), $G(x)$ can be extended continuously across infinity. The rest is well known. 


\section{REFERENCES}

[1] William K. Allard, On the first variation of a varifold, Ann. of Math.(2)95(1972),417-491.

[2] J. Cheeger and T.H. Colding, On the structure of spaces with Ricci curvature bounded below. I. J. Differential Geom. 46 (1997), no. 3, 406480.

[3] Shiing-shen Chern and R. Osserman, Complete minimal surfaces in euclidean n-space. J. Analyse Math. 19, 1967, 1534.

[4] T.H. Colding and W.P. Minicozzi II, The spaces of embedded minimal surfaces of fixed genus in a 3-manifold II; Multi-valued graphs in disks. Ann. of Math. 160(2004), 69-92.

[5] T.H. Colding and W.P. Minicozzi II, A Course in Minimal Surfaces, Graduate Studies in Mathematics 121, American Mathematical Society, Providence, RI (2011).

[6] P. Collin, Topologie et courbure des surfaces minimals proprement plonges de $\mathbb{R}^{3}$. Ann, of Math. (145)2, 1997, 1-31.

[7] C.J. Costa, Example of a complete minimal immersion in $\mathbb{R}^{3}$ of genus one and three embedded ends. Bol. Soc. Brasil. Mat. 15 (1984), no. 1-2, 4754

[8] C.J. Costa, Uniqueness of minimal surfaces embedded in $\mathbb{R}^{3}$ with total curvature 12 J. Differential Geom. 30 (1989), no. 3, 597618.

[9] C.J. Costa, Classification of complete minimal surfaces in $\mathbb{R}^{3}$ with total curvature $12 \pi$. Inventiones mathematicae,(105)1, 1991,273-303.

[10] Q. Chen, On the total curvature and area growth of minimal surfaces in $\mathbb{R}^{n}$. manuscipta math, 92, 135-142, 1997.

[11] G. David; C. Kenig and T. Toro, Asymptotically optimally doubling measures and Reifenberg flat sets with vanishing constant. Comm. Pure Appl. Math. 54 (2001), no. 4, 385449

[12] T. De Pauw, Nearly Flat Almost Monotone Measures are Big Pieces of Lipschitz Graphs. J. Geom. Anal. 12 no. 1, 2961,(2002).

[13] T. De Pauw, Concentrated, nearly monotonic, epiperimetric measures in Euclidean space. J. Differential Geom. 77, no. 1, 77134, 2007.

[14] H. Federer and W.H. Fleming, Normal and integral currents. Ann. of Math.(2)72(1960), 458-520.

[15] F. Hélein, Regularity of weakly harmonic maps from a surface into a manifold with symmetries, Manuscripta Math. 70(1991), 203-218.

[16] D. Hoffman and Wiliam H. Meeks, III, Embedded minimal surfaces of finite topology. Ann. of Math. (2) 131 (1990), no. 1, 134.

[17] A. Huber, On subharmonic functions and differential geometry in the large, Comment. Math. Helv. 32(1957) 181-206.

[18] T. Ilmanen, Singularities of Mean curvature flow of surfaces preliminary version(unpublished), 1995.

[19] H. Karcher. Embedded minimal surfaces derived from Scherk's examples. Manuscripta Math. (62) 83-114, 1988.

[20] E. Kuwert and Y.X. Li, $W^{2,2}$-conformal immersions of a closed Riemann surface into $\mathbb{R}^{n}$. Comm. Anal. Geom. 20 (2012), no. 2, 313340.

[21] E. Kuwert; Y.X. Li and R. Schätzle, The large genus limit of the infimum of the Willmore energy. Amer. J. Math. 132 (2010), no. 1, 3751.

[22] E. Kuwert and R.Schätzle, Removability of point singularities of Willmore surfaces. Annals of Mathematics, 2004, 160(1):315-357.

[23] Francisco J. Lopez, The Classification of Complete Minimal Surfaces with Total Curvature Greater Than $-12 \pi$. Transactions of the American Mathematical Society, (334) 1, 1992, 49-74.

[24] Francisco J. Lopez and A. Ros, On embedded complete minimal surfaces of genus zero, J. Diff. Geomm. (33), 1991, 293-300.

[25] William H. Meeks, Geometric results in classical minimal surface theory, volume 8 of Surveys in Differential Geometry. International Press, edited by S.T.Yau, 2003.

[26] William H. Meeks, III and Harold Rosenberg, The geometry and conformal structure of properly embedded minimal surfaces of finite topology in $\mathbb{R}^{3}$. Invent. Math. 114 (1993), no. 3,625639 .

[27] Wiliam H. Meeks, III and Michael Wolf, Minimal surfaces with area growth of two planes: The case of infinite symmetry, J. Amer. Math. Soc. 20 (2007), no. 2, 441465. 
[28] C.B. Morrey Jr., Multiple integrals in the calculus of variations. Die Grundlehren der mathematischen Wissenschaften, 130. Springer, New York, 1966.

[29] R.Osserman, Global properties of minimal surfaces in $E^{3}$ and $E^{n}$, Ann. of Math. (80)2, 340-364, 1964.

[30] R. Osserman, A survey of minimal surfaces, (2nd ed.), Dover, New York, 1986

[31] E.R. Reifenberg. Solution of the Plateau Problem for $\mathrm{m}$-dimensional surfaces of varying topological type. Acta Math. 1041960 192. (Reviewer: W. H. Fleming) 49.00

[32] J. Sacks and K. Uhlenbeck, The existence of minimal immersions of 2-Spheres. 113, 1-24, 1981.

[33] H.F. Scherk. Bemerkungen über die kleinste Fläche innerhalb gegebener Grenzen. J. R. Angew. Math., (13)185-208, 1835.

[34] R. Schoen. Uniqueness, Symmetry, and Embeddedness of minimal surface. Journal of Differential Geometry. 18, 1983,791-809.

[35] S. Semmes, Chord-arc surfaces with small constant. I. Adv. Math. 85 (1991), no. 2, 198223.

[36] S. Semmes, Chord-arc surfaces with small constant. II. Good parameterizations. Adv. Math. 88 (1991), no. 2, 170199.

[37] S. Semmes, Hypersurfaces in $\mathbb{R}^{n}$ whose unit normal has small BMO norm. Proc. Amer. Math. Soc. 112 (1991), no. 2, 403412.

[38] L. Simon, Existence of surfaces minimizing the Willmore functional. Comm. Anal. Geom. 1 (1993), no. 2, 281326.

[39] L. Simon, Introduction to Geometric Measure Theory. Tsinghua Lectures 2014

[40] L. Simon, Lectures on Geometric Measure Theory, Proc. Centre Math. Anal. Austral. Nat. Univ. 1983.

[41] L. Simon, Asymptotics for a class of nonlinear evolution equations, with applications to geometric problems. Ann. of Math. (2) 118 (1983), no. 3, 525571.

[42] L. Simon, Isolated singularities of extrema of geometric variational problems. Lecture Notes Math., Vol. 1161, pp. 206-277. Berlin Heidelberg New York: Springer 1985

[43] L.Simon. Reifenberg's Topological Disk Theorem. Mathematisches Institut Universitä t Tbingen Preprints AB Analysis, Preprint May 1996.

[44] J.X. Sun and J. Zhou, A Gromov-Hausdorff convergence theorem of surfaces in $\mathbb{R}^{n}$ with small total curvature, preprint.

[45] Emanuele Paolini, Regularity for minimal boundaries in $\mathbb{R}^{n}$ with mean curvature in $L^{n}$, manuscipta math. 97, 15-35, 1998.

Department of Mathematical Sciences, Tsinghua University, Beijing, P. R. China, 100084

Email: zhoujiemath@mail.tsinghua.edu.cn 